University of Windsor

Scholarship at UWindsor

2018

\title{
Consensus decision models for preferential voting with abstentions
}

Zaiwu Gong

Nanjing University of Information Science and Technology

Ning Zhang

Nanjing University of Information Science and Technology

Kevin Li

University of Windsor

Luis Martinez

Universidad de Jaén

Wei Zhao

Nanjing University of Information Science and Technology

Follow this and additional works at: https://scholar.uwindsor.ca/odettepub

Part of the Business Commons

\section{Recommended Citation}

Gong, Zaiwu; Zhang, Ning; Li, Kevin; Martinez, Luis; and Zhao, Wei. (2018). Consensus decision models for preferential voting with abstentions. Computers and Industrial Engineering, 115, 670-682.

https://scholar.uwindsor.ca/odettepub/136

This Article is brought to you for free and open access by the Odette School of Business at Scholarship at UWindsor. It has been accepted for inclusion in Odette School of Business Publications by an authorized administrator of Scholarship at UWindsor. For more information, please contact scholarship@uwindsor.ca. 
Computers and Industrial Engineering, 115: 670-682, 2018.

1 Consensus decision models for preferential voting with abstentions

Zaiwu Gong ${ }^{a}$, Ning Zhang ${ }^{b}$, Kevin W. Li $^{c^{*}}$, Luis Martínez ${ }^{d}$, Wei Zhao ${ }^{b}$

${ }^{a}$ Collaborative Innovation Center on Forecast and Evaluation of Meteorological Disasters, School of

4 Economics and Management, Nanjing University of Information Science and Technology, Nanjing 210044, China

${ }^{b}$ School of Economics and Management, Nanjing University of Information Science and Technology, Nanjing 210044, China

${ }^{c}$ Odette School of Business, University of Windsor, Windsor, Ontario N9B 3P4, Canada

${ }^{d}$ School of Computing, Universidad de Jaén, Campus Las Lagunillas s/n 23071, Spain

\section{Acknowledgments}

14 This research is partially supported by the National Natural Science Foundation of China 15 (Grant \#: 71171115, 71571104, 71572040), the Reform Foundation of Postgraduate 16 Education and Teaching in Jiangsu Province (Grant \#: JGKT10034), a Qing Lan Project 17 funded by the Priority Academic Program Development of Jiangsu Higher Education

18 Institutions, Natural Science Foundation of Jiangsu Province (Grant \#: BK20141481), a 19 Six Talent Peaks Project in Jiangsu Province (Grant \#: 2014-JY-014), a Discovery Grant 20 by the Natural Sciences and Engineering Research Council of Canada.

\footnotetext{
*Corresponding author, Telephone: 1 (519) 253-3000 ext 3456. Email: kwli@uwindsor.ca.
} 


\section{Highlights}

- A DEA model is proposed for preferential voting with abstentions.

- Raw votes are expressed as intervals with the width characterizing uncertain votes.

- The DEA model does not consider the influence of a moderator.

- Another model incorporates the influence of a moderator on uncertain votes.

- Economic significance is investigated for the second model and its dual models. 


\section{Abstract}

Proper use of the data envelopment analysis (DEA) for aggregating preferential rankings helps improve efficiency of a voting system. It has been observed that many recent elections often have low turnouts, a large number of abstentions and invalid ballots. If these voters can be influenced to cast their votes for or against a candidate, it is understandable that the voting result can be quite different. The purpose of this research is to incorporate abstentions into preferential voting models. To this end, we first introduce a preferential voting DEA model with abstentions, in which the raw votes are expressed as interval values and the width of the interval characterizes the number of uncertain votes, the objective function is to maximize a candidate's weighted voting score, and the constraints put restrictions on the place weights to ensure a proper importance order of different places. Secondly, given the fact that opinion leaders often employ different means such as social media and advertisement to influence voters in real-world elections, we explicitly incorporate these opinion leaders/brokers as a moderator into a preferential voting model with abstentions and introduce a moderator-involved-consensus preferential voting (MICPV) model. This model aims to capture the moderator's influences on the uncertain voters from a consensus perspective. The optimal allocation of all uncertain votes allows the moderator to maximize his/her influence over the voters to achieve the minimum deviation between his/her expected and the aggregate scores of the candidates. At the optimality, for those candidates where a complete consensus is achievable, the model identifies the optimal allocation scheme. We also analyze the economic significance of the MICPV model.

Keywords: Group decision making, Consensus, Preferential voting, Data envelopment analysis (DEA).

\section{Introduction}

In the industrial engineering discipline, decision making plays a key role in properly managing complex social, economic, and engineering systems such as group decision support (Beruvides, 1995), product development partner selection (Büyüközkan and Güleryüz, 2016), and ballot system design (Sivagami et al., 2011). Group decision-making (GDM) (Arrow, 1950; Fishburn, 1970) is the process of aggregating multiple decision-makers' (DMs') preferences over different alternatives into a compromised collective preference order for an entire group according to some rules (Hwang and Lin, 2012). GDM has a number of advantages: It helps to obtain more complete information and knowledge from different perspectives, generate more feasible alternatives, improve the group's acceptance of alterna- 
tives, and enhance the legitimacy of the decision making process. Thus, GDM can be a valuable tool to avoid individual irrational decisions caused by "bounded rationality" (Simon, 1991), risk aversion or risk appetite (Kahneman and Tversky, 1979). Based on a Bayesian approach, Altuzarra et al. (2010) constructed an AHP-group decision model for the consensus reaching process. On the other hand, GDM is not immune to pitfalls. For instance, it may lead to dangerous tendencies such as "groupthink" (Janis, 1972), "group polarization" (Stoner, 1961), and "escalation of commitment" (Myers and Lamm, 1975). To counter this downside risk, an experienced moderator with a global view of the issue at hand may be introduced to help avert these tendencies and improve decision results and the consensus reaching process (Palomares et al., 2014; Tan et al., 2017a,b; Zhang et al., 2017; Gong and Wang, 2017).

A preferential election is a special GDM process where multiple individuals participate in the voting process (decision analysis), establish decision (voting) rules, select appropriate methods for aggregating individual decisions (information aggregation), and choose a satisfactory alternative for the group (alternative selection). Three main processes are involved in a preferential election:

(1) Aggregating the voting information. Information aggregation is expected to satisfy the systematicity and integrity requirements of voting systems by exploiting different methods such as aggregation operators (i.e., weighted average operators), regression analysis, and function simulations (González-Pachón and Romero, 1999; Hummel, 2011; Bouton et al., 2015).

(2) Constructing voting rules. Efficient voting rules must be adaptable to different voting scenarios and help to improve the fairness of the voting process (Chandrasekher, 2015; Burnett and Kogan, 2015; Procaccia et al., 2016).

(3) Optimizing voting alternatives (i.e., voting system efficiency). An efficient voting system is expected to maximize the use of voting information, to obtain the best result based on an optimization model (Angiz et al., 2012; Gans and Smart, 1996; Tavares, 2012).

Generally speaking, the value of a voting system can be measured by its efficiency. The DEA model is a well known tool for evaluating efficiency because it takes a systematic approach to aggregate each candidate's score by incorporating the voting system information to optimize variable weights and ensure the rationality of voting ranks. As such, Cook and Kress (1990) proposed to use a DEA model to aggregate preferential ranking with a focus on the efficiency of a voting system. The research question therein is to select $k$ members out of $m$ candidates and rank them according to the importance of their places. Subsequently, Cook et al. (1997) presented a general distance-based consensus framework for deriving ordinal ranking. To solve the problem of preference ranking, Wang et al. $(2005,2007)$ put forward an interval utility function and ordered weight averaging operators. Gong et al. (2015a) constructed consensus models to deal with interval preference opinions and interpreted their economic significance. Franceschini and Maisano (2015) introduced a new quantitative tool to check the consistency of the solution in ordinal semi-democratic decision-making problems. By 
integrating interval 2-tuple linguistic TOPSIS into DEA, Geng at al. (2016) investigated a component oriented remanufacturing decision-making problem. Over the past 20 years, voting efficiency also attracted significant interests from many researchers (Oukil and Amin, 2015; Ebrahimnejad et al., 2016; Llamazares and Peӥa, 2009; Sun and Ma, 2015).

In recent elections, low turnout ratios have been a consistent and notorious problem. To increase turnouts, different measures have been proposed. For instance, some jurisdictions have experimented with mandatory voting. It has been observed that these measures often result in a large number of invalid ballots and abstentions (Power and Roberts, 1995). It is understandable that these votes may have a significant effect on the voting result depending upon whether they can be converted to approval votes. For instance, we often observe different social media conduct polls prior to the final preferential voting and predict who will win/lose the election. In their questionnaires, undecided is typically one of the choices for potential voters who take the survey. These undecided votes can be conveniently characterized as interval-valued voting counts. During the campaign, the candidates can then use the poll results to adjust their running strategies and launch advertisements to influence those undecided voters. Our proposed models can be of use for the runners and their managers in allocating their campaign resources to maximize their chance of winning over these uncertain voters. Building upon Borda's (1784) voting method and Cook and Kress (1990)'s DEA model, this paper firstly proposes a novel preferential voting paradigm to incorporate abstentions (hereafter, invalid ballots are treated as abstentions in this article) by introducing interval values for the raw vote counts where the interval width measures the magnitude of abstention votes. Furthermore, since voting efficiency evaluation aims to adequately aggregate the voting information, the derived result presumably represents the collective choice of the voters. From this perspective, the voting process can be treated as a consensus decision problem, which is an active research topic in the industrial engineering field. For instance, recent studies examined consistency and consensus with different types of decision input such as hesitant fuzzy linguistic preference relations (Wu and Xu, 2015; Wang and Gong, 2017), interval fuzzy preference relations (Meng et al., 2016), and heterogeneous preference relations (Chen et al., 2015). Labella et al. (2017) conducted a comparative analysis of the performance of classical consensus models in large-scale GDM.

Contemporary elections have presented a common scenario in which different lobby groups, think tanks, and opinion leaders express their opinions on the candidates and some may even launch campaigns to promote their preferred candidate and/or undermine others. Their goal is to influence voters so that their preferred candidate can be elected as the winner. To properly consider their role in an election, a second proposal is presented in this paper that explicitly introduces them into a consensus decision model (Liu et al., 2017; Wu et al., 2018, 2017) and collectively refers them to as a moderator (Ben-Arieh and Easton, 2007; Herrera-Viedma et al., 2007; Gong et al., 2015a). Consistent with the idea in (Dong et al., 2018), where a moderator can strategically manipulate attribute weights or 
allocate resources to obtain her/his desired alternatives' ranking, this second novel approach, a socalled moderator-involved consensus preferential voting (MICPV) model, aims to achieve consensus between the moderator's expectations and the voters' aggregate voting scores of the candidates by minimizing their deviations. This type of research and treatment has been widely used in industrial engineering and engineering management. We also explore the economic significance of different model components in MICPV and its dual models using the primal-dual linear programming theory.

Key features of both proposed models are:

(a) Abstentions are allowed and accommodated by allowing raw voting counts to assume interval values. Without loss of generality, invalid ballots are treated as abstentions in this article.

(b) The place weights differ and are functions of a discriminating factor.

(c) The objective function of the primal model minimizes the difference between the moderator's expected scores and the weighted voting scores for all candidates. The purpose is to reach the highest possible consensus between the moderator's opinion and those of the voters.

(d) The objective function of the dual model aims to measure the total utility that the moderator's opinion influences the voting scores of the candidates.

The rest of this paper is structured as follows. Section 2 introduces Cook and Kress's DEA model for aggregating preference rankings. Section 3 presents a generalization of the DEA model allowing for abstentions. Section 4 proposes an MICPV model with abstentions. Different primal and dual linear programs are also discussed for the consensus reaching process. Section 5 explores economic significance of key model components. Illustrative examples are provided in Section 6 to analyze a hypothetical preferential voting scenario with and without a moderator's influence. The paper concludes with some remarks in Section 7.

\section{Background}

In Cook and Kress (1990)'s DEA model, there exist $k$ places in a preferential election. Each voter must select $k$ winners from $m$ candidates in a preferential order of their places. Assume that the vote of candidate $i$ in place $j$ is $v_{i j}, i \in M=\{1, \ldots, m\}, j \in K=\{1, \ldots, k\}$, and the importance of place $j$ is $\omega_{j}$. Then, the candidate $i$ 's overall score is $W_{i}=\sum_{j=1}^{k} \omega_{j} v_{i j}$. Specifically, for a particular candidate $i_{0} \in M$, to determine an optimal set of $\left\{\omega_{j}^{*}\right\}$ that maximizes his/her weighted score $W_{i_{0}}$, Cook and Kress (1990) proposed to employ the following DEA model for aggregating preference ranking (DEA 
voting model):

$$
\begin{aligned}
& \operatorname{Max} W_{i_{0}}=\sum_{j=1}^{k} \omega_{j} v_{i_{0} j} \\
& \text { s.t. }\left\{\begin{array}{l}
\sum_{j=1}^{k} \omega_{j} v_{i j} \leq 1, i \in M \\
\left.\omega_{j}-\omega_{j+1} \geq d(j, \varepsilon), j=1, \ldots, k-1\right) \\
\omega_{k} \geq d(k, \varepsilon)
\end{array} \quad(1-2)\right.
\end{aligned}
$$

Constraint (1-1) indicates that the upper bound for any candidate $i$ 's score is 1 . Constraints (12 ) and (1-3) put restrictions on the weights of the places. The importance of the places are listed in a descending order, where the lower bound $d(j, \varepsilon)$ on the gap between the importance of two successive places is referred to as the discrimination intensity function. The parameter $\varepsilon$ is called the discrimination factor. Assume that $d(j, \varepsilon)=f(j) \varepsilon$ is a monotonically non-decreasing linear function, and $f(j)$ is a positive constant with respect to $j$. The objective function is candidate $i_{0}$ 's weighted voting score (hereinafter referred to as score) $\sum_{j=1}^{k} \omega_{j} v_{i_{0} j}$, which is to be maximized.

The characteristics of the DEA voting model can be briefly summarized as follows:

(a) The place weights are objectively determined as an optimal solution to the DEA voting model.

(b) The differences in importance of places are modeled as constraints in a linear programming model, which is computationally easier to solve compared to a nonlinear model.

(c) The candidate's final score is computed as a linear weighted sum based on the optimal weights derived from the DEA model.

Cook and Kress's original DEA model assumes that all votes are valid without abstentions. But as it is noted in Section 1, a large number of invalid ballots and abstentions often arise in elections. To make the aforesaid DEA model more applicable, Section 3 proposes a preferential voting DEA model with abstentions. The voting score of candidate $i$ in place $j, v_{i j}$, assumes an interval value, where the interval width (distance between the lower and the upper bounds of the interval) is the maximum number of invalid votes or abstentions. The optimal weight of candidate $i$ in place $j$ is obtained by using a DEA method, thereby determining the final candidate ranking.

\section{A preferential voting DEA model with abstentions}

In a preferential election with abstentions, $k$ places are vacant. Each voter selects $k$ winners from $m$ candidates and ranks them accordingly. Assume the voting score interval of candidate $i$ in place $j$ is $\left[v_{i j}^{L}, v_{i j}^{U}\right], i \in M, j \in K . v_{i j}^{L}$ and $v_{i j}^{U}$ denote the lower and upper bounds of votes for candidate $i$ in place $j$. The interval $\left[v_{i j}^{L}, v_{i j}^{U}\right]$ can be viewed as a crisp number $v_{i j}^{L}+\alpha_{i j}\left(v_{i j}^{U}-v_{i j}^{L}\right)$, where $0 \leq \alpha_{i j} \leq 1 . v_{i j}^{U}-v_{i j}^{L}$ is the number of uncertain votes, which may be valid abstentions or invalid ballots. Understandably, at 
an aggregate level, voters often have different opinions toward the same candidate in different places, and the number of uncertain votes (or interval widths) for the same candidate can vary considerably for distinct places. For instance, if the interval voting score for candidate 1 in place 1 is $[2,6]$, then at least 2 and at most 6 voters vote candidate 1 in place 1; while candidate 1 obtains an interval voting score of $[1,7]$ in place 2 , then at least 1 and at most 7 voters vote the same candidate in place 2 . When $\alpha_{i j}=0$, none of the uncertain votes is treated as approval of candidate $i$ in place $j$, providing the minimum votes $v_{i j}^{L}$. Conversely, when $\alpha_{i j}=1$, all uncertain votes are viewed as approvals of candidate $i$ in place $j$, providing the upper bound of possible votes $v_{i j}^{U}$. Thus, $\alpha_{i j}$ indicates the allocation scheme of uncertain votes for candidate $i$ in place $j$, and the adjusted number of votes is $v_{i j}^{L}+\alpha_{i j}\left(v_{i j}^{U}-v_{i j}^{L}\right)$ after uncertain votes are reallocated. As $\alpha_{i j}$ represents how the uncertain votes are reallocated as approval votes for candidate $i$ in place $j$, for the same candidate $i$, the allocation schemes $\alpha_{i j}$ are independently determined for different places $j$ 's and $\alpha_{i 1}, \alpha_{i 2}$, and $\alpha_{i k}$ are independent of each other. Additionally, the importance of place $j$ is denoted by $\omega_{j}$, and the weighted score of candidate $i$ is given by $\sum_{j=1}^{k} \omega_{j}\left[v_{i j}^{L}+\alpha_{i j}\left(v_{i j}^{U}-v_{i j}^{L}\right)\right]$. A preferential voting DEA model with abstentions is thus proposed as

$$
\begin{aligned}
& Z_{i_{0}}(\varepsilon)=\operatorname{Max} \sum_{j=1}^{k} \omega_{j}\left[v_{i_{0} j}^{L}+\alpha_{i_{0} j}\left(v_{i_{0} j}^{U}-v_{i_{0} j}^{L}\right)\right] \\
& \text { s.t. } \begin{cases}\sum_{j=1}^{k} \omega_{j}\left[v_{i j}^{L}+\alpha_{i j}\left(v_{i j}^{U}-v_{i j}^{L}\right)\right] \leq 1, i \in M & (2-1) \\
\omega_{j}-\omega_{j+1} \geq d(j, \varepsilon), j=1,2, \cdots, k-1 & (2-2) \\
\omega_{k} \geq d(k, \varepsilon) & (2-3) \\
0 \leq \alpha_{i j} \leq 1, i \in M, j \in K & (2-4)\end{cases}
\end{aligned}
$$

The $k$ place weights $\left(\omega_{k}, k \in K\right)$ can be objectively obtained using Model (2). Then, candidate $i_{0}$ 's overall score is calculated as a weighted average of the optimal interval values $\left(\left[v_{i_{0} j}^{L}, v_{i_{0} j}^{U}\right]\right)$. A final ranking for all candidates can then be determined by their overall scores.

Model (2) is nonlinear. Let $m_{i j} \triangleq \omega_{j} \alpha_{i j}$, as $0 \leq \alpha_{i j} \leq 1$, we have $0 \leq m_{i j} \leq \omega_{j}$, then Model (2) is transformed into linear programming Model (3) as follows:

$$
\begin{aligned}
& Z_{i_{0}}(\varepsilon)=\operatorname{Max} \sum_{j=1}^{k}\left[\omega_{j} v_{i_{0} j}^{L}+m_{i_{0} j}\left(v_{i_{0} j}^{U}-v_{i_{0} j}^{L}\right)\right] \\
& \text { s.t. } \begin{cases}\sum_{j=1}^{k}\left[\omega_{j} v_{i j}^{L}+m_{i j}\left(v_{i j}^{U}-v_{i j}^{L}\right)\right] \leq 1, i \in M & (3-1) \\
\omega_{j}-\omega_{j+1} \geq d(j, \varepsilon), j=1,2, \cdots, k-1 & (3-2) \\
\omega_{k} \geq d(k, \varepsilon) & (3-3) \\
0 \leq m_{i j} \leq \omega_{j}, i \in M, j \in K & (3-4)\end{cases}
\end{aligned}
$$

Theorem 1. For any $i_{0} \in M, Z_{i_{0}}(\varepsilon)$ is a monotonically non-increasing function of $\varepsilon$.

Proof. For any given $\varepsilon$ and $\varepsilon^{\prime}, \varepsilon^{\prime} \geq \varepsilon \geq 0$, as $d\left(j, \varepsilon^{\prime}\right)=f(j) \varepsilon^{\prime}$ and $d(j, \varepsilon)=f(j) \varepsilon$, and $f(j)>0$, we have $d\left(j, \varepsilon^{\prime}\right) \geq d(j, \varepsilon)$. Let $\varphi(\varepsilon)$ and $\varphi\left(\varepsilon^{\prime}\right)$ be the feasible region of Model (3). Then, it implies that $\varphi\left(\varepsilon^{\prime}\right) \subseteq \varphi(\varepsilon)$. Therefore, $Z_{i_{0}}\left(\varepsilon^{\prime}\right) \leq Z_{i_{0}}(\varepsilon)$. 
Theorem 1 shows that the smaller the discrimination factor $\varepsilon$, the bigger the candidate's overall score, i.e., reducing the discrimination factor decreases the gaps among the place weights and makes places more competitive. For a particular candidate, places with more votes tend to be assigned heavier weights, thereby increasing the candidate's overall score.

Corollary 1. If $Z_{i_{0}}\left(\varepsilon^{\prime}\right)=1$, then $Z_{i_{0}}(\varepsilon)=1$ for all $\varepsilon \leq \varepsilon^{\prime}$.

Proof. $\varepsilon \leq \varepsilon^{\prime}$ and $Z_{i_{0}}(\varepsilon)$ is a monotonically non-increasing function, so $Z_{i_{0}}(\varepsilon) \geq Z_{i_{0}}\left(\varepsilon^{\prime}\right)=1$. From Constraint (3-1), one has $Z_{i_{0}}(\varepsilon) \leq 1$. As such $Z_{i_{0}}(\varepsilon)=1$.

Equivalently, for $\varepsilon, \varepsilon^{\prime} \geq 0$, if $Z_{i_{0}}\left(\varepsilon^{\prime}\right)=1$ and $Z_{i_{0}}(\varepsilon)<1$, then $\varepsilon>\varepsilon^{\prime}$.

Corollary 1 shows a higher discrimination factor increases the differences between place weights and makes the places less competitive. In this case, places with relatively high votes tend to be assigned smaller weights, thereby decreasing the candidate's weighted score.

For any $i \in M$, let $\varepsilon_{\text {max }}^{*}$ be the largest value of $\varepsilon$ such that $Z_{i}(\varepsilon)=1, \varepsilon_{\text {max }}^{*}=\operatorname{Max}_{i}\left\{\varepsilon_{i}^{*}\right\}$, where $\varepsilon_{i}^{*}=\operatorname{Max}\left\{\varepsilon \mid Z_{i}(\varepsilon)=1\right\}$.

Theorem 2. For $\forall \varepsilon \in\left[0, \varepsilon_{\text {max }}^{*}\right]$, there exists a candidate $i_{0}$ such that $Z_{i_{0}}(\varepsilon) \geq Z_{i}(\varepsilon), \forall i \in M$.

Proof. Let $\varepsilon_{i_{0}}^{*} \triangleq \varepsilon_{\text {max }}^{*}$, then $Z_{i_{0}}(\varepsilon)=1$ holds for any $\varepsilon \in\left[0, \varepsilon_{\max }^{*}\right]$. For any candidate $i$, if $\varepsilon \leq \varepsilon_{i}^{*} \leq \varepsilon_{\text {max }}^{*}, Z_{i}(\varepsilon)=1$; if $\varepsilon_{i}^{*} \leq \varepsilon \leq \varepsilon_{\max }^{*}, Z_{i}(\varepsilon)<1$.

In conclusion, $Z_{i_{0}}(\varepsilon) \geq Z_{i}(\varepsilon)$ holds for $\varepsilon \in\left[0, \varepsilon_{\text {max }}^{*}\right]$.

If the discrimination factor $\varepsilon$ falls within a certain range, Theorem 2 demonstrates that there must exist a candidate who achieves the highest score, implying that Model (3) can always find a winning candidate.

Theorem 3. $\varepsilon_{\max }^{*}$ is the largest value of $\varepsilon$ such that Model (3) is still feasible.

Proof. Without loss of generality, assume that $\varepsilon_{\text {max }}^{*}<\infty$. We prove this theorem by contradiction. Let $\varepsilon>\varepsilon_{\text {max }}^{*}$, and there exists a feasible solution $\omega=\left(w_{1}, \ldots, \omega_{k}\right)^{T}$ to Model $(3)$ with a discrimination factor $\varepsilon$. According to the definition of $\varepsilon_{\text {max }}^{*}$, we have $Z_{i}(\varepsilon)<1$ for all $i \in M$. The dual of primal Model (3) is

$$
\begin{aligned}
& H_{i_{0}}(\varepsilon)=\operatorname{Min} \sum_{i=1}^{m} x_{i}-\sum_{j=1}^{k} y_{j} d(j, \varepsilon) \\
& \text { s.t. } \begin{cases}\sum_{i=1}^{m}\left(v_{i 1}^{L} x_{i}-n_{i 1}\right)-y_{1} \geq v_{i_{0}}^{L} \\
\sum_{i=1}^{m}\left(v_{i j}^{L} x_{i}-n_{i j}\right)+y_{j-1}-y_{j} \geq v_{i_{0} j}^{L}, j=2,3, \cdots, k & (4-2) \\
\left(v_{i j}^{U}-v_{i j}^{L}\right) x_{i}+n_{i j} \geq 0, i \neq i_{0}, i \in M, j \in K \\
\left(v_{i_{0} j}^{U}-v_{i_{0} j}^{L}\right) x_{i}+n_{i_{0} j} \geq v_{i_{0} j}^{U}-v_{i_{0} j}^{L}, j \in K \\
x_{i}, y_{j}, n_{i j} \geq 0, i \in M, j \in K\end{cases}
\end{aligned}
$$

From the principle of complementary slackness, the optimal solution to Model (4) is $x_{1}^{*}=\cdots=$ $x_{m}^{*}=0$, which contradicts (4-1) or (4-2). This completes the proof.

The contrapositive of Theorem 3: If there does not exist a feasible solution to Model (3), then 
the discrimination factor $\varepsilon$ exceeds the maximum $\varepsilon_{\max }^{*}$, implying that the difference between place weights $f(j) \varepsilon_{\max }^{*}$ exceeds an upper bound.

Theorem 3 establishes an upper bound $\varepsilon_{\max }^{*}$ for the discrimination factor such that a feasible solution exists for Model (3). Model (3) will not be able to identify the best candidate if the discrimination factor exceeds $\varepsilon_{\max }^{*}$ (i.e., the difference between two successive place weights exceeds a certain threshold).

Clearly, the discrimination factor affects the place weights and ultimately influences candidates' scores. Therefore, it is convenient to explore how sensitive place weights and the optimal choice of the candidate are affected by the discrimination factor. Additionally, at the maximum discrimination factor $\varepsilon_{\max }$, two drawbacks of the Cook and Kress DEA model can be overcome: (i) different candidates will receive distinct scores and (ii) unified place weights will be derived for all candidates. Thus, a preferential voting model with abstentions is introduced below to maximize the discrimination factor:

$$
\begin{aligned}
& \operatorname{Max} \varepsilon \\
& \text { s.t. }\left\{\begin{array}{ll}
\sum_{j=1}^{k}\left[\omega_{j} v_{i j}^{L}+m_{i j}\left(v_{i j}^{U}-v_{i j}^{L}\right)\right] \leq 1, i \in M & (5-1) \\
\omega_{j}-\omega_{j+1}-d(j, \varepsilon) \geq 0, j=1,2, \cdots, k-1 & (5-2) \\
\omega_{k}-d(k, \varepsilon) \geq 0 & (5-3) \\
0 \leq m_{i j} \leq \omega_{j}, i \in M, j \in K \\
\omega_{j}, \varepsilon \geq 0, j \in K
\end{array}\right)(5-4)
\end{aligned}
$$

Theorem 4. For an optimal solution to Model (5), at least one of the constraints in (5-1) is binding and holds as an equality.

Proof. According to Theorem 3, the optimal objective value of Model (5) is $\varepsilon_{\text {max }}^{*}$, which satisfies all the constraints. Assume that $\sum_{j=1}^{k}\left[\omega_{j} v_{i j}^{L}+m_{i j}\left(v_{i j}^{U}-v_{i j}^{L}\right)\right]<1$ holds for all $i \in M$ under the optimal solution to Model (5), its dual model is given as

$$
\begin{aligned}
& \operatorname{Min} \sum_{i=1}^{m} x_{i} \\
& \text { s.t. } \begin{cases}\sum_{i=1}^{m}\left(v_{i 1}^{L} x_{i}-n_{i 1}\right)-y_{1} \geq 0 & (6-1) \\
\sum_{i=1}^{m}\left(v_{i j}^{L} x_{i}-n_{i j}\right)+y_{j-1}-y_{j} \geq 0, j=2,3, \cdots, k & (6-2) \\
\left(v_{i j}^{U}-v_{i j}^{L}\right) x_{i}+n_{i j} \geq 0, i \in M, j \in K & (6-3) \\
\sum_{j=1}^{k} y_{j} \geq 1, j=2,3, \cdots, k & (6-4) \\
x_{i}, y_{j}, n_{i j} \geq 0, i \in M, j \in K & (6-5)\end{cases}
\end{aligned}
$$

Based on the principle of complementary slackness, the optimal solution to Model $(6)$ is $x_{1}^{*}=$ $\cdots=x_{m}^{*}=0$. Then, either (6-1) or (6-2) in Model (6) is violated. Therefore, there exists $i_{0}$ such that $\sum_{j=1}^{k}\left[\omega_{j} v_{i_{0} j}^{L}+m_{i_{0} j}\left(v_{i_{0} j}^{U}-v_{i_{0} j}^{L}\right)\right]=1$. 
Theorem 4 indicates that the optimal discrimination factor guarantees the existence of a candidate with the highest score in a voting system with abstentions.

Theorem 5. Under the optimal solution to Model (5), all the constraints in (5-2) and (5-3) are binding.

Proof. Denote Model (5)'s optimal solution by

$$
\left(\omega_{1}^{*}, \ldots, \omega_{k}^{*}, m_{11}^{*}, \ldots, m_{m 1}^{*}, \ldots, m_{1 j}^{*}, \ldots, m_{m j}^{*}, \ldots, m_{1 k}^{*}, \ldots, m_{m k}^{*}, \varepsilon_{\max }^{*}\right)^{T} .
$$

Let $\omega^{*}=\left(\omega_{1}^{*}, \ldots, \omega_{k}^{*}\right)^{T}$, we shall prove this theorem by contradiction. Suppose that $\omega_{s}^{*}-\omega_{s+1}^{*}-$ $d\left(s, \varepsilon_{\max }^{*}\right)=\Gamma>0$ for some $s$. Define

$$
\omega_{j}^{\prime}= \begin{cases}\omega_{j}^{*}-\Gamma, & j=1,2, \cdots, s \\ \omega_{j}^{*}, & j=s+1, \cdots, k\end{cases}
$$

Since $m_{i j}=\omega_{j} \alpha_{i j}$ and $0 \leq \alpha_{i j} \leq 1$, one has

$$
m_{i j}^{\prime}= \begin{cases}m_{i j}^{*}-\alpha_{i j}^{*} \Gamma, & j=1,2, \cdots, s \\ m_{i j}^{*}, & j=s+1, \cdots, k\end{cases}
$$

Let $I_{0}=\left\{i \mid \sum_{j=1}^{k}\left[\omega_{j}^{*} v_{i j}^{L}+m_{i j}^{*}\left(v_{i j}^{U}-v_{i j}^{L}\right)\right]=1\right\}$ and, without loss of generality, assume that $v_{i j}^{U}>0$ for all $i \in I_{0}$. For $\forall i \in I_{0}$, if $i \in\left\{i \mid v_{i j}^{L} \neq 0\right\}$, we have

$$
\begin{gathered}
\sum_{j=1}^{k}\left[\omega_{j}^{\prime} v_{i j}^{L}+m_{i j}^{\prime}\left(v_{i j}^{U}-v_{i j}^{L}\right)\right] \\
<\sum_{j=1}^{k}\left[\omega_{j}^{*} v_{i j}^{L}+m_{i j}^{*}\left(v_{i j}^{U}-v_{i j}^{L}\right)\right]=1
\end{gathered}
$$

If there exists at least an $i \in\left\{i \mid v_{i j}^{L}=0, i \in I_{0}\right\}$, then there must exist one $j_{0}$ such that $m_{i j_{0}}^{*}=$ $\omega_{j_{0}}^{*} \alpha_{i j_{0}}^{*} \neq 0$ and

- if $j_{0} \in\{1,2, \ldots, s\}$, then $m_{i j_{0}}^{\prime}=\left(\omega_{j_{0}}^{*}-\Gamma\right) \alpha_{i j_{0}}^{*} \neq 0$.

- if $j_{0} \in\{s+1, \ldots, k\}$, for $m_{i j_{0}}^{*}=\omega_{j_{0}}^{*} \alpha_{i j_{0}}^{*}>0$, then $\omega_{j_{0}}^{*}>0$. In the meantime, as $\omega_{s}^{*}-\omega_{s+1}^{*}-$ $d\left(s, \varepsilon_{\max }^{*}\right)=\Gamma>0$, then $\omega_{j}^{*} \geq \Gamma$ for $j \in\{1,2, \ldots, s\}$. Therefore, there must exist a $j_{1}$ such that $\omega_{j_{1}}^{*}>\Gamma$ for $j_{1} \in\{1,2, \ldots, s-1\}$. Thus we have $m_{i j_{1}}^{\prime}=\left(\omega_{j_{1}}^{*}-\Gamma\right) \alpha_{i j_{1}}^{*} \neq 0$.

In sum, there must exist one $j$ such that $m_{i j}^{\prime}=\left(\omega_{j}^{*}-\Gamma\right) \alpha_{i j}^{*} \neq 0$ for $j \in\{1,2, \ldots, s\}$. Clearly,

$$
\begin{aligned}
& \sum_{j=1}^{k}\left[\omega_{j}^{\prime} v_{i j}^{L}+m_{i j}^{\prime}\left(v_{i j}^{U}-v_{i j}^{L}\right)\right] \\
= & \sum_{j=1}^{s}\left[\omega_{j}^{\prime} v_{i j}^{L}+m_{i j}^{\prime}\left(v_{i j}^{U}-v_{i j}^{L}\right)\right]+\sum_{j=s+1}^{k}\left[\omega_{j}^{*} v_{i j}^{L}+m_{i j}^{*}\left(v_{i j}^{U}-v_{i j}^{L}\right)\right] \\
< & \sum_{j=1}^{k}\left[\omega_{j}^{*} v_{i j}^{L}+m_{i j}^{*}\left(v_{i j}^{U}-v_{i j}^{L}\right)\right]=1
\end{aligned}
$$


From Eqs. (7) and (8), if $\forall i \in I_{0}$, we have

$$
\begin{gathered}
\sum_{j=1}^{k}\left[\omega_{j}^{\prime} v_{i j}^{L}+m_{i j}^{\prime}\left(v_{i j}^{U}-v_{i j}^{L}\right)\right] \\
<\sum_{j=1}^{k}\left[\omega_{j}^{*} v_{i j}^{L}+m_{i j}^{*}\left(v_{i j}^{U}-v_{i j}^{L}\right)\right]=1
\end{gathered}
$$

If $\forall i \notin I_{0}$, we have

$$
\begin{gathered}
\sum_{j=1}^{k}\left[\omega_{j}^{\prime} v_{i j}^{L}+m_{i j}^{\prime}\left(v_{i j}^{U}-v_{i j}^{L}\right)\right] \\
<\sum_{j=1}^{k}\left[\omega_{j}^{*} v_{i j}^{L}+m_{i j}^{*}\left(v_{i j}^{U}-v_{i j}^{L}\right)\right]<1
\end{gathered}
$$

$\omega_{j}^{\prime}-\omega_{j+1}^{\prime}-d\left(j, \varepsilon_{\max }^{*}\right) \geq 0$, and $\omega_{s}^{\prime}-\omega_{s+1}^{\prime}-d\left(j, \varepsilon_{\max }^{*}\right)=0$.

Thus, $\left(\omega_{1}^{\prime}, \ldots, \omega_{k}^{\prime}, m_{11}^{\prime}, \ldots, m_{m 1}^{\prime}, \ldots, m_{1 j}^{\prime}, \ldots, m_{m j}^{\prime}, \ldots, m_{1 k}^{\prime}, \ldots, m_{m k}^{\prime}\right)^{T}$ is also an optimal solution to Model (5).

Consequently, from Eqs. (9) and (10), we conclude that all constraints in (5-1) are non-binding, which contradicts Theorem 4 .

Theorem 5 demonstrates that, when the discrimination factor is maximized, the place weight differences reach the lower bound of the discrimination intensity function. In this case, different candidates receive distinct scores. Thus, Model (6) can be viewed as an effective evaluation model for ranking candidates.

\section{A moderator-involved-consensus preferential voting model with abstentions}

Section 3 extends Cook and Kress's preferential voting DEA model (1990) to allow for abstentions by assuming interval values for candidates' raw votes. The model assumes that each voter casts his/her ballot on his/her own consideration without external influences. However, in many elections around the globe nowadays, it is typical that influential opinion leaders publish their preferences on different candidates and try to influence the voters' decision. These opinion leaders usually represent certain interests and are often knowledgeable about the issues at hand. They may exploit their resources to launch campaigns on different media to support their preferred candidate and undermine competitors before the voters go to the poll to cast their ballots. It is understandable that their purpose is to influence the voters' opinion so that their preferred candidate can receive the best voting score. In this section, we shall extend the model in Section 3 by explicitly considering the role of opinion leaders in preferential voting. This idea is consistent with the notion of leader's role in opinion dynamics in (Dong et al., 2017). Following the terminology used in (Ben-Arieh and Easton, 2007; Herrera-Viedma et al., 2007; Gong et al., 2015a,b), opinion leaders are referred to as a moderator hereafter (Palomares et al., 2014). From the moderator's perspective, he/she expects that the voters consider both their 
individual and his/her opinions. His/her objective is to reach a consensus between the voters and him/herself so that his/her expected ranking can be realized or approximated as much as possible through the voting process. In the proposed MICPV model, the objective is to minimize the deviation between the moderator's expected score and the weighted voting score for each candidate subject to various constraints on place weights.

In the proposed model, it is assumed that the moderator's expected score for candidate $i$ is $o_{i}$, $i \in M$, and the interval value of the votes for candidate $i$ in place $j$ is $\left[v_{i j}^{L}, v_{i j}^{U}\right], i \in M, j \in K$. For instance, $o_{1}=7$ means the moderator's expected score for candidate 1 is 7 . Similar to Section $3, v_{i j}^{L}$ and $v_{i j}^{U}$ denote the lower and upper bounds of votes for candidate $i$ in place $j$ and the interval $\left[v_{i j}^{L}, v_{i j}^{U}\right]$ can be transformed into a crisp number $v_{i j}^{L}+\alpha_{i j}\left(v_{i j}^{U}-v_{i j}^{L}\right)$, where $0 \leq \alpha_{i j} \leq 1$ and can be interpreted in the same way as before. Under the influence of the moderator, the voting result tends to come closer to the expected score of the moderator. The closer the candidate $i$ 's overall weighted score $S_{i}\left(\omega_{j}, \alpha_{i j}\right)=$ $\sum_{j=1}^{k} \omega_{j}\left[v_{i j}^{L}+\alpha_{i j}\left(v_{i j}^{U}-v_{i j}^{L}\right)\right]$ to $o_{i}$ (i.e., the deviation $d_{i}\left(\omega_{j}, \alpha_{i j}\right)=\left|\sum_{j=1}^{k} \omega_{j}\left[v_{i j}^{L}+\alpha_{i j}\left(v_{i j}^{U}-v_{i j}^{L}\right)\right]-o_{i}\right|$ is small), the better the voting result obtained by the moderator in terms of his/her expectation.

In other words, the moderator can adjust the importance (or weights) of the places to influence the voters, thereby making the voting score $\sum_{j=1}^{k} \omega_{j}\left[v_{i j}^{L}+\alpha_{i j}\left(v_{i j}^{U}-v_{i j}^{L}\right)\right]$ approach $o_{i}$, and achieving the best voting consensus. Therefore, an MICPV model with abstentions is formulated as

$$
\begin{aligned}
& \operatorname{Min} W(\varepsilon)=\sum_{i=1}^{m}\left|\sum_{j=1}^{k} \omega_{j}\left[v_{i j}^{L}+\alpha_{i j}\left(v_{i j}^{U}-v_{i j}^{L}\right)\right]-o_{i}\right| \\
& \text { s.t. } \begin{cases}\omega_{j}-\omega_{j+1} \geq d(j, \varepsilon), j=1, \ldots, k-1 & (11-1) \\
\omega_{k} \geq d(k, \varepsilon) & (11-2) \\
0 \leq \alpha_{i j} \leq 1, i \in M, j \in K & (11-3)\end{cases}
\end{aligned}
$$

In Model (11), the objective function can be interpreted as the overall consensus degree between the moderator's expectation and all voters' aggregate voting scores for all candidates. A smaller $\sum_{i=1}^{m} d_{i}\left(\omega_{j}, \alpha_{i j}\right)$ corresponds to a higher consensus. The place weights $\omega_{j}$ satisfy constraints $(11-1)-(11-$ $3)$.

Let $m_{i j} \triangleq \omega_{j} \alpha_{i j}$. As $0 \leq \alpha_{i j} \leq 1$, we have $0 \leq m_{i j} \leq \omega_{j}$. Let $a_{i} \triangleq \frac{1}{2}\left\{\left[\mid \sum_{j=1}^{k}\left[\omega_{j} v_{i j}^{L}+m_{i j}\left(v_{i j}^{U}-v_{i j}^{L}\right)\right]-\right.\right.$ $\left.\left.o_{i} \mid+\left(\sum_{j=1}^{k}\left[\omega_{j} v_{i j}^{L}+m_{i j}\left(v_{i j}^{U}-v_{i j}^{L}\right)\right]-o_{i}\right)\right]\right\}$, and $b_{i} \triangleq \frac{1}{2}\left\{\left[\left|\sum_{j=1}^{k}\left[\omega_{j} v_{i j}^{L}+m_{i j}\left(v_{i j}^{U}-v_{i j}^{L}\right)\right]-o_{i}\right|-\left(\sum_{j=1}^{k}\left[\omega_{j} v_{i j}^{L}+m_{i j}\left(v_{i j}^{U}-\right.\right.\right.\right.\right.$ $\left.\left.\left.\left.\left.v_{i j}^{L}\right)\right]-o_{i}\right)\right]\right\}$. One has $a_{i}+b_{i}=\left|\sum_{j=1}^{k}\left[\omega_{j} v_{i j}^{L}+m_{i j}\left(v_{i j}^{U}-v_{i j}^{L}\right)\right]-o_{i}\right|, a_{i}-b_{i}=\sum_{j=1}^{k}\left[\omega_{j} v_{i j}^{L}+m_{i j}\left(v_{i j}^{U}-v_{i j}^{L}\right)\right]-o_{i}$. 
Thus, Model (11) is equivalent to the following linear programming model:

$$
\begin{aligned}
& \text { Min } W(\varepsilon)=\sum_{i=1}^{m}\left(a_{i}+b_{i}\right) \\
& \text { s.t. } \begin{cases}\sum_{j=1}^{k}\left[\omega_{j} v_{i j}^{L}+m_{i j}\left(v_{i j}^{U}-v_{i j}^{L}\right)\right]-a_{i}+b_{i}=o_{i}, i \in M & (12-1) \\
\omega_{j}-\omega_{j+1} \geq d(j, \varepsilon), j=1, \ldots, k-1 & (12-2) \\
\omega_{k} \geq d(k, \varepsilon) & (12-3) \\
\omega_{j} \geq m_{i j}, i \in M, j \in K & (12-4) \\
a_{i}, b_{i}, m_{i j} \geq 0, i \in M, j \in K & (12-5)\end{cases}
\end{aligned}
$$

Theorem 6. $W(\varepsilon)$ is a monotonically non-decreasing function of $\varepsilon$.

Proof. For any given $\varepsilon$ and $\varepsilon^{\prime}, \varepsilon^{\prime} \geq \varepsilon \geq 0$, let $\varphi(\varepsilon), \varphi\left(\varepsilon^{\prime}\right)$, respectively, be the feasible region of Model (12) corresponding to $\varepsilon$ and $\varepsilon^{\prime}$, it is clear $\varphi\left(\varepsilon^{\prime}\right) \subseteq \varphi(\varepsilon)$. Thus, $W\left(\varepsilon^{\prime}\right) \geq W(\varepsilon)$.

Recall that the smaller the objective function in Model (12), the higher the consensus level. Theorem 6 shows that the consensus level increases as the discrimination factor decreases. This article assumes that $d(j, \varepsilon)=f(j) \varepsilon$, where $f(j)$ is a nonnegative constant. So, a reduction in $\varepsilon$ leads to smaller gaps among the place weights. Therefore, the consensus level can be improved by reducing $\varepsilon$ or the differences between place weights. Since $\varepsilon$ affects the consensus level, the following three scenarios are considered for the place weight constraints in the MICPV model with abstentions.

1. Nonnegative place weight constraints $\left(\omega_{j} \geq 0, \varepsilon=0, j \in K\right)$;

2. Lower bound place weight constraints $\left(\omega_{j} \geq \varepsilon, \varepsilon>0, j \in K\right)$ and;

3. Descending place weight constraints $\left(\omega_{j}-\omega_{j+1} \geq d(j, \varepsilon), j=1, \ldots, k-1, \omega_{k} \geq d(k, \varepsilon)\right)$.

Specific properties of these three cases and their dual models are established next in Sections 4.1-4.3.

\subsection{An MICPV Model with Abstentions under Nonnegative Place Weight Constraints}

With nonnegative place weight constraints, Model (11) can be simplified to

$$
\begin{aligned}
& \operatorname{Min} \sum_{i=1}^{m}\left|\sum_{j=1}^{k} \omega_{j}\left[v_{i j}^{L}+\alpha_{i j}\left(v_{i j}^{U}-v_{i j}^{L}\right)\right]-o_{i}\right| \\
& \text { s.t. }\left\{\begin{array}{l}
\omega_{j} \geq 0, j \in K \\
0 \leq \alpha_{i j} \leq 1, i \in M, j \in K
\end{array}\right.
\end{aligned}
$$

Let $m_{i j} \triangleq \omega_{j} \alpha_{i j}$. Model (13) is converted to an equivalent linear programming Model (14):

$$
\begin{aligned}
& \operatorname{Min} \sum_{i=1}^{m}\left(a_{i}+b_{i}\right) \\
& \text { s.t. }\left\{\begin{array}{l}
\sum_{j=1}^{k}\left[\omega_{j} v_{i j}^{L}+m_{i j}\left(v_{i j}^{U}-v_{i j}^{L}\right)\right]-a_{i}+b_{i}=o_{i}, i \in M \\
\omega_{j} \geq m_{i j}, i \in M, j \in K \\
a_{i}, b_{i}, \omega_{j}, m_{i j} \geq 0, i \in M, j \in K
\end{array}\right.
\end{aligned}
$$


The dual of Model (14) is

$$
\begin{aligned}
& \operatorname{Max} \sum_{i=1}^{m} o_{i} y_{i} \\
& \text { s.t. }\left\{\begin{array}{l}
\sum_{i=1}^{m}\left(v_{i j}^{L} y_{i}+n_{i j}\right) \leq 0, j \in K \\
\left(v_{i j}^{U}-v_{i j}^{L}\right) y_{i}-n_{i j} \leq 0, i \in M, j \in K \\
-1 \leq y_{i} \leq 1, i \in M \\
n_{i j} \geq 0, i \in M, j \in K
\end{array}\right.
\end{aligned}
$$

The following theorems or properties are mainly derived by the principle of complementary slackness from the primal-dual linear programming theory. They are useful in our economic significance interpretations of MICPV models with abstentions in Section 5.

Corollary 2. When $\varepsilon=0, \sum_{i=1}^{m}\left\{\sum_{j=1}^{k}\left[\omega_{j} v_{i j}^{L}+m_{i j}\left(v_{i j}^{U}-v_{i j}^{L}\right)\right]\right\} y_{i}=0$.

Proof. It is understandable that each place weight satisfies $\omega_{j}>0$. According to the principle of complementary slackness, $\sum_{i=1}^{m}\left(v_{i j}^{L} y_{i}+n_{i j}\right)=0$ holds for $\forall j \in K$. Thus,

$$
\sum_{i=1}^{m} \sum_{j=1}^{k}\left(\omega_{j} v_{i j}^{L} y_{i}+\omega_{j} n_{i j}\right)=0
$$

Denote $A_{1} \triangleq\left\{(i, j) \mid \alpha_{i j}=0\right\}, A_{2} \triangleq\left\{(i, j) \mid \alpha_{i j}=1\right\}$, and $A_{3} \triangleq\left\{(i, j) \mid 0<\alpha_{i j}<1\right\}$. Obviously, $A_{1} \cup A_{2} \cup A_{3}=M \times K$.

- When $(i, j) \in A_{1}$. As $m_{i j}=0$ and $\omega_{j}-m_{i j}>0$, we have $n_{i j}=0$. On the other hand, $m_{i j}\left(v_{i j}^{U}-v_{i j}^{L}\right) y_{i}=0$, so $m_{i j}\left(v_{i j}^{U}-v_{i j}^{L}\right) y_{i}-\omega_{j} n_{i j}=0$.

- When $(i, j) \in A_{2}$. Since $m_{i j}=\omega_{j}>0$, we have $\left(v_{i j}^{U}-v_{i j}^{L}\right) y_{i}-n_{i j}=0$. Therefore, $m_{i j}\left[\left(v_{i j}^{U}-\right.\right.$ $\left.\left.v_{i j}^{L}\right) y_{i}-n_{i j}\right]=m_{i j}\left(v_{i j}^{U}-v_{i j}^{L}\right) y_{i}-m_{i j} n_{i j}=m_{i j}\left(v_{i j}^{U}-v_{i j}^{L}\right) y_{i}-\omega_{j} n_{i j}=0$.

- When $(i, j) \in A_{3}$. As $m_{i j}=\omega_{j} \alpha_{i j}>0$ and $\omega_{j}-m_{i j}>0$, we have $\left(v_{i j}^{U}-v_{i j}^{L}\right) y_{i}-n_{i j}=0$ and $n_{i j}=0$. Thus, $\left(v_{i j}^{U}-v_{i j}^{L}\right) y_{i}=0$. As such, one has $m_{i j}\left(v_{i j}^{U}-v_{i j}^{L}\right) y_{i}-\omega_{j} n_{i j}=0$.

In summary, $m_{i j}\left(v_{i j}^{U}-v_{i j}^{L}\right) y_{i}-\omega_{j} n_{i j}=0$ holds for $\forall i \in M, j \in K$. Therefore,

$$
\sum_{i=1}^{m} \sum_{j=1}^{k}\left[m_{i j}\left(v_{i j}^{U}-v_{i j}^{L}\right) y_{i}-\omega_{j} n_{i j}\right]=0
$$

From Eqs. (16) and (17), we have $\sum_{i=1}^{m} \sum_{j=1}^{k}\left[\omega_{j} v_{i j}^{L} y_{i}+m_{i j}\left(v_{i j}^{U}-v_{i j}^{L}\right) y_{i}\right]=0$. Thus, $\sum_{i=1}^{m}\left\{\sum_{j=1}^{k}\left[\omega_{j} v_{i j}^{L}+\right.\right.$ $\left.\left.m_{i j}\left(v_{i j}^{U}-v_{i j}^{L}\right)\right]\right\} y_{i}=0$.

Let $I_{0} \triangleq\left\{i \mid o_{i}=\sum_{j=1}^{k}\left[\omega_{j} v_{i j}^{L}+m_{i j}\left(v_{i j}^{U}-v_{i j}^{L}\right)\right]\right\}, I_{1} \triangleq\left\{i \mid o_{i}>\sum_{j=1}^{k}\left[\omega_{j} v_{i j}^{L}+m_{i j}\left(v_{i j}^{U}-v_{i j}^{L}\right)\right]\right\}$, and $I_{2} \triangleq$ $\left\{i \mid o_{i}<\sum_{j=1}^{k}\left[\omega_{j} v_{i j}^{L}+m_{i j}\left(v_{i j}^{U}-v_{i j}^{L}\right)\right]\right\}$, then $I_{0} \cup I_{1} \cup I_{2}=M$.

Corollary 3. For $i \in I_{1}, y_{i}=1$; for $i \in I_{2}, y_{i}=-1$. 
Proof. In Model (14), if $i \in I_{1}$ (i.e., $\left.o_{i}>\sum_{j=1}^{k}\left[\omega_{j} v_{i j}^{L}+m_{i j}\left(v_{i j}^{U}-v_{i j}^{L}\right)\right]\right), a_{i}=0$ and $b_{i}>0$. According to the complementary slackness property, $y_{i}=1$. If $i \in I_{2}$, we can similarly show that $y_{i}=-1$.

Theorem 7. Let $w_{j}^{*}, m_{i j}^{*}$ be the optimal solution to Model (14), if $I_{0}=\emptyset$ then $\sum_{i \in I_{1}} \sum_{j=1}^{k}\left[\omega_{j}^{*} v_{i j}^{L}+\right.$ $\left.m_{i j}^{*}\left(v_{i j}^{U}-v_{i j}^{L}\right)\right]=\sum_{i \in I_{2}} \sum_{j=1}^{k}\left[\omega_{j}^{*} v_{i j}^{L}+m_{i j}^{*}\left(v_{i j}^{U}-v_{i j}^{L}\right)\right]$.

Proof. If $I_{0}=\emptyset, I_{1} \cup I_{2}=M$. According to Corollary 2, $\sum_{i=1}^{m}\left\{\sum_{j=1}^{k}\left[\omega_{j} v_{i j}^{L}+m_{i j}\left(v_{i j}^{U}-v_{i j}^{L}\right)\right]\right\} y_{i}=$ $\sum_{i \in I_{1}}\left\{\sum_{j=1}^{k}\left[\omega_{j} v_{i j}^{L}+m_{i j}\left(v_{i j}^{U}-v_{i j}^{L}\right)\right]\right\} y_{i}+\sum_{i \in I_{2}}\left\{\sum_{j=1}^{k}\left[\omega_{j} v_{i j}^{L}+m_{i j}\left(v_{i j}^{U}-v_{i j}^{L}\right)\right]\right\} y_{i}=0$. As per Corollary 3 , when $i \in I_{1}$, $y_{i}=1$; and when $i \in I_{2}, y_{i}=-1$. So $\sum_{i \in I_{1}} \sum_{j=1}^{k}\left[\omega_{j}^{*} v_{i j}^{L}+m_{i j}^{*}\left(v_{i j}^{U}-v_{i j}^{L}\right)\right]=\sum_{i \in I_{2}} \sum_{j=1}^{k}\left[\omega_{j}^{*} v_{i j}^{L}+m_{i j}^{*}\left(v_{i j}^{U}-v_{i j}^{L}\right)\right]$.

Theorem 7 implies that, if the moderator's opinion always differs from the candidates' aggregate voting scores, then the candidates' uncertain scores will be evenly split into two parts, where the sum of the underachieved scores obtained by all candidates in $I_{1}$ is equal to that of the overachieved scores in $I_{2}$.

Theorem 8. Let $\omega_{j}^{*}, m_{i j}^{*}$ be the optimal solution to Model (14). Dual Model (15) is equivalent to:

$$
\begin{aligned}
& \operatorname{Max} \sum_{i=1}^{m}\left\{o_{i}-\sum_{j=1}^{k}\left[\omega_{j}^{*} v_{i j}^{L}+m_{i j}^{*}\left(v_{i j}^{U}-v_{i j}^{L}\right)\right]\right\} y_{i} \\
& \text { s.t. }\left\{\begin{array}{l}
\sum_{i=1}^{m}\left(v_{i j}^{L} y_{i}+n_{i j}\right) \leq 0, j \in K \\
\left(v_{i j}^{U}-v_{i j}^{L}\right) y_{i}-n_{i j} \leq 0, i \in M, j \in K \\
-1 \leq y_{i} \leq 1, i \in M \\
n_{i j} \geq 0, i \in M, j \in K
\end{array}\right.
\end{aligned}
$$

Proof. From Corollary 2, we have $\sum_{i=1}^{m}\left\{\sum_{j=1}^{k}\left[\omega_{j}^{*} v_{i j}^{L}+m_{i j}^{*}\left(v_{i j}^{U}-v_{i j}^{L}\right)\right]\right\} y_{i}=0$.

From the primal and dual models (14) and (18), one can prove that the voting intervals incorporating abstentions have the following three properties.

Let $I^{+} \triangleq\left\{i \mid y_{i}>0\right\}, I^{-} \triangleq\left\{i \mid y_{i}<0\right\}$, and $I^{0} \triangleq\left\{i \mid y_{i}=0\right\}$.

Property 1. If $i \in I^{+}, \alpha_{i j}=1$.

Proof. If $i \in I^{+}, y_{i}>0$. If $v_{i j}^{U}=v_{i j}^{L}, v_{i j}^{L}+\alpha_{i j}\left(v_{i j}^{U}-v_{i j}^{L}\right) \equiv v_{i j}^{L}$, which implies that there are no abstentions for placing candidate $i$ at $j$, in this case, it is irrelevant what value $\alpha_{i j}$ assumes and we can simply let $\alpha_{i j}=1$. Otherwise, as $\left(v_{i j}^{U}-v_{i j}^{L}\right) y_{i}-n_{i j} \leq 0$, one has $n_{i j}>0$. According to the principle of complementary slackness, we have $\omega_{j}-m_{i j}=0$ and $\alpha_{i j}=1$.

Property 1 indicates that when $y_{i}>0$, all abstentions of candidate $i$ in place $j$ are treated as approval votes.

Property 2. If $i \in I^{-}, \alpha_{i j}=0$.

Proof. When $i \in I^{-}$(i.e., $\left.y_{i}<0\right)$, we have $\left(v_{i j}^{U}-v_{i j}^{L}\right) y_{i}-n_{i j}<0$. According to the principle of complementary slackness, we have $m_{i j}=0$ and $\alpha_{i j}=0$. 
Property 2 implies that, when $y_{i}<0$, none of the abstentions of candidate $i$ in place $j$ is treated as approval votes.

Property 3. If $i \in I^{0}, a_{i}=b_{i}=0$ and $0<\alpha_{i j}<1$.

Proof. If $i \in I^{0}, y_{i}=0$. Since $-1<y_{i}<1$, according to the principle of complementary slackness, we have $a_{i}=b_{i}=0$. As such, $0<\alpha_{i j}<1$.

Property 3 shows that, if $y_{i}=0$, a complete consensus between the moderator's expectation and the aggregate score for candidate $i$ can be achieved by properly allocating all uncertain votes (i.e., adjusting the value of $\alpha_{i j}$ ).

\subsection{An MICPV Model with Abstentions under Lower Bound Place Weight Constraints}

Under lower bound place weight constraints, Model (11) can be simplified to

$$
\begin{aligned}
& \operatorname{Min} \sum_{i=1}^{m}\left|\sum_{j=1}^{k} \omega_{j}\left[v_{i j}^{L}+\alpha_{i j}\left(v_{i j}^{U}-v_{i j}^{L}\right)\right]-o_{i}\right| \\
& \text { s.t. }\left\{\begin{array}{l}
\omega_{j} \geq \varepsilon, j \in K \\
0 \leq \alpha_{i j} \leq 1, i \in M, j \in K
\end{array}\right.
\end{aligned}
$$

Let $m_{i j} \triangleq \omega_{j} \alpha_{i j}$, Model (19) is equivalent to the linear programming Model (20):

$$
\begin{aligned}
& \operatorname{Min} \sum_{i=1}^{m}\left(a_{i}+b_{i}\right) \\
& \text { s.t. }\left\{\begin{array}{l}
\sum_{j=1}^{k}\left[\omega_{j} v_{i j}^{L}+m_{i j}\left(v_{i j}^{U}-v_{i j}^{L}\right)\right]-a_{i}+b_{i}=o_{i}, i \in M \\
\omega_{j} \geq m_{i j}, i \in M, j \in K \\
\omega_{j} \geq \varepsilon, j \in K \\
a_{i}, b_{i}, m_{i j} \geq 0, i \in M, j \in K
\end{array}\right.
\end{aligned}
$$

Let $z_{j} \triangleq \omega_{j}-\varepsilon$, Model (20) can be transformed to

$$
\begin{aligned}
& \operatorname{Min} \sum_{i=1}^{m}\left(a_{i}+b_{i}\right) \\
& \text { s.t. }\left\{\begin{array}{l}
\sum_{j=1}^{k}\left[z_{j} v_{i j}^{L}+m_{i j}\left(v_{i j}^{U}-v_{i j}^{L}\right)\right]-a_{i}+b_{i}=o_{i}-\varepsilon \sum_{j=1}^{k} v_{i j}^{L}, i \in M \\
z_{j}-m_{i j} \geq-\varepsilon, i \in M, j \in K \\
a_{i}, b_{i}, z_{j}, m_{i j} \geq 0, i \in M, j \in K
\end{array}\right.
\end{aligned}
$$

The dual of Model (21) is

$$
\begin{aligned}
& \operatorname{Max} \sum_{i=1}^{m}\left(o_{i}-\varepsilon \sum_{j=1}^{k} v_{i j}^{L}\right) h_{i}-\varepsilon \sum_{i=1}^{m} \sum_{j=1}^{k} p_{i j} \\
& \text { s.t. }\left\{\begin{array}{l}
\sum_{i=1}^{m}\left(v_{i j}^{L} h_{i}+p_{i j}\right) \leq 0, j \in K \\
\left(v_{i j}^{U}-v_{i j}^{L}\right) h_{i}-p_{i j} \leq 0, i \in M, j \in K \\
-1 \leq h_{i} \leq 1, i \in M \\
p_{i j} \geq 0, i \in M, j \in K
\end{array}\right.
\end{aligned}
$$


The following results are derived based on the principle of complementary slackness in the primaldual linear programming theory.

Corollary 4. If $\varepsilon>0, \sum_{i=1}^{m}\left\{\sum_{j=1}^{k}\left[\omega_{j} v_{i j}^{L}+m_{i j}\left(v_{i j}^{U}-v_{i j}^{L}\right)\right]\right\} h_{i}=\sum_{i=1}^{m} \sum_{j=1}^{k} \varepsilon v_{i j}^{L} h_{i}+\sum_{i=1}^{m} \sum_{j=1}^{k} \varepsilon p_{i j}$.

Proof. It is clear that the place weights satisfy $\omega_{j}>0$ for all $j \in K$. According to complementary slackness, $\sum_{i=1}^{m}\left(v_{i j}^{L} h_{i}+p_{i j}\right)=0$ holds for $\forall j \in K$. As such, $z_{j} \sum_{i=1}^{m}\left(v_{i j}^{L} h_{i}+p_{i j}\right)=\sum_{i=1}^{m}\left(z_{j} v_{i j}^{L} h_{i}+z_{j} p_{i j}\right)=0$. Thus,

$$
\sum_{i=1}^{m} \sum_{j=1}^{k}\left(z_{j} v_{i j}^{L} h_{i}+z_{j} p_{i j}\right)=0
$$

Let $A_{1} \triangleq\left\{(i, j) \mid \alpha_{i j}=0\right\}, A_{2} \triangleq\left\{(i, j) \mid \alpha_{i j}=1\right\}$, and $A_{3} \triangleq\left\{(i, j) \mid 0<\alpha_{i j}<1\right\}$. Obviously, $A_{1} \cup A_{2} \cup A_{3}=M \times K$.

- $(i, j) \in A_{1}$ : In this case, $m_{i j}=\omega_{j} \alpha_{i j}=0$ and $z_{j}-m_{i j}>-\varepsilon$, we have $p_{i j}=0$. Thus, $m_{i j}\left(v_{i j}^{U}-v_{i j}^{L}\right) h_{i}-\left(z_{j}+\varepsilon\right) p_{i j}=0$ holds.

- $(i, j) \in A_{2}$ : Here $m_{i j}=\omega_{j}=z_{j}+\varepsilon>0$, we have $\left(v_{i j}^{U}-v_{i j}^{L}\right) h_{i}-p_{i j}=0$. Thus, $m_{i j}\left(v_{i j}^{U}-v_{i j}^{L}\right) h_{i}-$ $\left(z_{j}+\varepsilon\right) \omega_{j} p_{i j}=0$ holds.

- $(i, j) \in A_{3}$ : As $m_{i j}=\omega_{j} \alpha_{i j}>0$ and $z_{j}-m_{i j}>-\varepsilon$, we have $\left(v_{i j}^{U}-v_{i j}^{L}\right) h_{i}-p_{i j}=0$ and $p_{i j}=0$, implying that $\left(v_{i j}^{U}-v_{i j}^{L}\right) h_{i}=0$. Then, $m_{i j}\left(v_{i j}^{U}-v_{i j}^{L}\right) h_{i}-\left(z_{j}+\varepsilon\right) p_{i j}=0$ holds.

Combining the aforesaid three cases, $m_{i j}\left(v_{i j}^{U}-v_{i j}^{L}\right) h_{i}-\left(z_{j}+\varepsilon\right) p_{i j}=0$ holds for $\forall i \in M, j \in K$. Therefore, we have

$$
\sum_{i=1}^{m} \sum_{j=1}^{k}\left[m_{i j}\left(v_{i j}^{U}-v_{i j}^{L}\right) h_{i}-\left(z_{j}+\varepsilon\right) p_{i j}\right]=0
$$

Adding Eqs. (23) and (24), we have $\sum_{i=1}^{m} \sum_{j=1}^{k}\left[z_{j} v_{i j}^{L} h_{i}+m_{i j}\left(v_{i j}^{U}-v_{i j}^{L}\right) h_{i}\right]=\sum_{i=1}^{m} \sum_{j=1}^{k} \varepsilon p_{i j}$. Plugging $z_{j}=\omega_{j}-\varepsilon$ into this equation and rearranging the terms, one has $\sum_{i=1}^{m}\left\{\sum_{j=1}^{k}\left[\omega_{j} v_{i j}^{L}+m_{i j}\left(v_{i j}^{U}-v_{i j}^{L}\right)\right]\right\} h_{i}=$ $\sum_{i=1}^{m} \sum_{j=1}^{k} \varepsilon v_{i j}^{L} h_{i}+\sum_{i=1}^{m} \sum_{j=1}^{k} \varepsilon p_{i j}$. Corollary 4 is thus proved.

Similarly, let $I_{0} \triangleq\left\{i \mid o_{i}=\sum_{j=1}^{k}\left[\omega_{j} v_{i j}^{L}+m_{i j}\left(v_{i j}^{U}-v_{i j}^{L}\right)\right]\right\}, I_{1} \triangleq\left\{i \mid o_{i}>\sum_{j=1}^{k}\left[\omega_{j} v_{i j}^{L}+m_{i j}\left(v_{i j}^{U}-v_{i j}^{L}\right)\right]\right\}$, and $I_{2} \triangleq\left\{i \mid o_{i}<\sum_{j=1}^{k}\left[\omega_{j} v_{i j}^{L}+m_{i j}\left(v_{i j}^{U}-v_{i j}^{L}\right)\right]\right\}$, then $I_{0} \cup I_{1} \cup I_{2}=M$.

Corollary 5. If $i \in I_{1}, h_{i}=1$ and if $i \in I_{2}, h_{i}=-1$.

Proof. In Model (20), if $i \in I_{1}$ (i.e., $\left.o_{i}>\sum_{j=1}^{k}\left[\omega_{j} v_{i j}^{L}+m_{i j}\left(v_{i j}^{U}-v_{i j}^{L}\right)\right]\right)$ we have $a_{i}=0$ and $b_{i}>0$. Based on the complementary slackness property, $h_{i}=1$. Similarly, if $i \in I_{2}, h_{i}=-1$. 
Theorem 9. Let $z_{j}^{*}, m_{i j}^{*}$ be the optimal solution to Model (21). Dual Model (22) is equivalent to:

$$
\begin{array}{ll}
\operatorname{Max} & \sum_{i=1}^{m}\left\{o_{i}-\sum_{j=1}^{k}\left[\omega_{j}^{*} v_{i j}^{L}+m_{i j}^{*}\left(v_{i j}^{U}-v_{i j}^{L}\right)\right]\right\} h_{i} \\
\text { s.t. }\left\{\begin{array}{l}
\sum_{i=1}^{m}\left(v_{i j}^{L} h_{i}+p_{i j}\right) \leq 0, j \in K \\
\left(v_{i j}^{U}-v_{i j}^{L}\right) h_{i}-p_{i j} \leq 0, i \in M, j \in K \\
-1 \leq h_{i} \leq 1, i \in M \\
p_{i j} \geq 0, i \in M, j \in K
\end{array}\right.
\end{array}
$$

where $\omega_{j}^{*}=z_{j}^{*}+\varepsilon$ is the optimal solution to Model (21).

Proof. From Corollary 4, we have $\sum_{i=1}^{m}\left\{\sum_{j=1}^{k}\left[\omega_{j}^{*} v_{i j}^{L}+m_{i j}^{*}\left(v_{i j}^{U}-v_{i j}^{L}\right)\right]\right\} h_{i}=\sum_{i=1}^{m} \sum_{j=1}^{k} \varepsilon v_{i j}^{L} h_{i}+\sum_{i=1}^{m} \sum_{j=1}^{k} \varepsilon p_{i j}$.

\subsection{An MICPV Model with Abstentions under Descending Place Weight Constraints}

This subsection presents an MICPV model with abstentions and descending place weight constraints $\omega_{j}-\omega_{j+1} \geq d(j, \varepsilon)$.

In this case, Model (11) is equivalent to Model (12) and the dual of Model (12) is:

$$
\begin{aligned}
& \operatorname{Max} \sum_{i=1}^{m} o_{i} d_{i}+\sum_{j=1}^{k} d(j, \varepsilon) x_{j} \\
& \text { s.t. } \begin{cases}\sum_{i=1}^{m}\left(v_{i 1}^{L} d_{i}+q_{i 1}\right)+x_{1} \leq 0 & (26-1) \\
\sum_{i=1}^{m}\left(v_{i j}^{L} d_{i}+q_{i j}\right)-x_{j-1}+x_{j} \leq 0, j=2, \ldots, k & (26-2) \\
\left(v_{i j}^{U}-v_{i j}^{L}\right) d_{i}-q_{i j} \leq 0, i \in M, j \in K & (26-3) \\
-1 \leq d_{i} \leq 1, i \in M & (26-4) \\
q_{i j}, x_{j} \geq 0, i \in M, j \in K & (26-5)\end{cases}
\end{aligned}
$$

In a similar fashion, as per complementary slackness in the primal-dual linear programming theory, we obtain the following results.

Corollary 6. $\sum_{i=1}^{m} \sum_{j=1}^{k}\left[\omega_{j} v_{i j}^{L}+m_{i j}\left(v_{i j}^{U}-v_{i j}^{L}\right)\right] d_{i}+\sum_{j=1}^{k} d(j, \varepsilon) x_{j}=0$.

Proof. Similarly, the place weights satisfy $\omega_{j}>0, j \in K$. By the principle of complementary slackness, $\sum_{i=1}^{m}\left(v_{i 1}^{L} d_{i}+q_{i 1}\right)+x_{1}=0$ and $\sum_{i=1}^{m}\left(v_{i j}^{L} d_{i}+q_{i j}\right)-x_{j-1}+x_{j}=0, j=2,3, \ldots, k$. Then, we have $\sum_{i=1}^{m}\left(v_{i 1}^{L} d_{i}+q_{i 1}\right) \omega_{1}+x_{1} \omega_{1}=0$ and $\sum_{i=1}^{m}\left(v_{i j}^{L} d_{i}+q_{i j}\right) \omega_{j}-x_{j-1} \omega_{j}+x_{j} \omega_{j}=0, j=2,3, \ldots, k$. Thus,

$$
\sum_{i=1}^{m} \sum_{j=1}^{k}\left(v_{i j}^{L} d_{i}+q_{i j}\right) \omega_{j}+\sum_{j=1}^{k-1}\left(\omega_{j}-\omega_{j+1}\right) x_{j}+\omega_{k} x_{k}=0
$$

Let $A_{1} \triangleq\left\{(i, j) \mid \alpha_{i j}=0\right\}, A_{2} \triangleq\left\{(i, j) \mid \alpha_{i j}=1\right\}$, and $A_{3} \triangleq\left\{(i, j) \mid 0<\alpha_{i j}<1\right\}$. Obviously, $A_{1} \cup A_{2} \cup A_{3}=M \times K$.

- $(i, j) \in A_{1}$ : In this case, $m_{i j}=0$ and $\omega_{j}-m_{i j}>0$, we have $q_{i j}=0$. Thus, $m_{i j}\left(v_{i j}^{U}-v_{i j}^{L}\right) d_{i}-\omega_{j} q_{i j}=$ 0 . 
- $(i, j) \in A_{2}$ : If $m_{i j}=\omega_{j}>0$, we have $\left(v_{i j}^{U}-v_{i j}^{L}\right) d_{i}-q_{i j}=0$. Thus, $m_{i j}\left(v_{i j}^{U}-v_{i j}^{L}\right) d_{i}-\omega_{j} q_{i j}=0$.

- $(i, j) \in A_{3}$ : Here, as $m_{i j}=\omega_{j} \alpha_{i j}>0$ and $\omega_{j}-m_{i j}>0$, we have $\left(v_{i j}^{U}-v_{i j}^{L}\right) d_{i}-q_{i j}=0$ and $q_{i j}=0$, meaning that $\left(v_{i j}^{U}-v_{i j}^{L}\right) d_{i}=0$. Thus, $m_{i j}\left(v_{i j}^{U}-v_{i j}^{L}\right) d_{i}-\omega_{j} q_{i j}=0$.

In sum, $m_{i j}\left(v_{i j}^{U}-v_{i j}^{L}\right) d_{i}-\omega_{j} q_{i j}=0$ holds for $\forall i \in M, j \in K$. Therefore,

$$
\sum_{i=1}^{m} \sum_{j=1}^{k}\left[m_{i j}\left(v_{i j}^{U}-v_{i j}^{L}\right) d_{i}-\omega_{j} q_{i j}\right]=0
$$

Adding Eqs. (27) and (28), we have

$$
\sum_{i=1}^{m} \sum_{j=1}^{k}\left[\omega_{j} v_{i j}^{L} d_{i}+m_{i j}\left(v_{i j}^{U}-v_{i j}^{L}\right) d_{i}\right]+\sum_{j=1}^{k-1}\left(\omega_{j}-\omega_{j+1}\right) x_{j}+\omega_{k} x_{k}=0
$$

- If $d(j, \varepsilon)=0$, in this case, $\omega_{j}-\omega_{j+1}>0, j=1,2, \cdots, k-1$ and $\omega_{k}>0$, we have $x_{j}=0$. Thus,

$$
\sum_{j=1}^{k-1}\left(\omega_{j}-\omega_{j+1}\right) x_{j}+\omega_{k} x_{k}=\sum_{j=1}^{k} d(j, \varepsilon) x_{j}
$$

- If $d(j, \varepsilon)>0$, denote $J_{1} \triangleq\left\{j \mid x_{j}=0, j \neq k\right\}$ and $J_{2} \triangleq\left\{j \mid x_{j}>0, j \neq k\right\}$.

$-j \in J_{1}:$ As $x_{j}=0$, we have $\sum_{j \in J_{1}}\left(\omega_{j}-\omega_{j+1}\right) x_{j}=\sum_{j \in J_{1}} d(j, \varepsilon) x_{j}$.

$-j \in J_{2}:$ Since $x_{j}>0$, we have $\omega_{j}-\omega_{j+1}=d(j, \varepsilon)$ and $\sum_{j \in J_{2}}\left(\omega_{j}-\omega_{j+1}\right) x_{j}=\sum_{j \in J_{2}} d(j, \varepsilon) x_{j}$.

Thus, we have $\sum_{j \in J_{1}}\left(\omega_{j}-\omega_{j+1}\right) x_{j}+\sum_{j \in J_{2}}\left(\omega_{j}-\omega_{j+1}\right) x_{j}=\sum_{j \in J_{1}} d(j, \varepsilon) x_{j}+\sum_{j \in J_{2}} d(j, \varepsilon) x_{j}$. Therefore,

$$
\sum_{j=1}^{k-1}\left(\omega_{j}-\omega_{j+1}\right) x_{j}=\sum_{j=1}^{k-1} d(j, \varepsilon) x_{j}
$$

We have two cases for $x_{k}$ :

- if $x_{k}=0$, we have $\omega_{k} x_{k}=0$ and $d(k, \varepsilon) x_{k}=0$, so $\omega_{k} x_{k}=d(k, \varepsilon) x_{k} ;$ and

- if $x_{k}>0$, we have $\omega_{k}=d(k, \varepsilon)$, so $\omega_{k} x_{k}=d(k, \varepsilon) x_{k}$.

Therefore,

$$
\omega_{k} x_{k}=d(k, \varepsilon) x_{k}
$$

Adding Eqs. (31) and (32), we have $\sum_{j=1}^{k-1}\left(\omega_{j}-\omega_{j+1}\right) x_{j}+\omega_{k} x_{k}=\sum_{j=1}^{k-1} d(j, \varepsilon) x_{j}+d(k, \varepsilon) x_{k}$, that is,

$$
\sum_{j=1}^{k-1}\left(\omega_{j}-\omega_{j+1}\right) x_{j}+\omega_{k} x_{k}=\sum_{j=1}^{k} d(j, \varepsilon) x_{j}, \text { if } d(j, \varepsilon)>0
$$

Combining Eqs. (30) and (33), we have

$$
\sum_{j=1}^{k-1}\left(\omega_{j}-\omega_{j+1}\right) x_{j}+\omega_{k} x_{k}=\sum_{j=1}^{k} d(j, \varepsilon) x_{j}, \text { if } d(j, \varepsilon) \geq 0
$$


Consequently, plugging (34) into (29) yields $\sum_{i=1}^{m} \sum_{j=1}^{k}\left[\omega_{j} v_{i j}^{L}+m_{i j}\left(v_{i j}^{U}-v_{i j}^{L}\right)\right] d_{i}+\sum_{j=1}^{k} d(j, \varepsilon) x_{j}=0$.

Let $I_{0} \triangleq\left\{i \mid o_{i}=\sum_{j=1}^{k}\left[\omega_{j} v_{i j}^{L}+m_{i j}\left(v_{i j}^{U}-v_{i j}^{L}\right)\right]\right\}, I_{1} \triangleq\left\{i \mid o_{i}>\sum_{j=1}^{k}\left[\omega_{j} v_{i j}^{L}+m_{i j}\left(v_{i j}^{U}-v_{i j}^{L}\right)\right]\right\}$, and $I_{2} \triangleq$ $\left\{i \mid o_{i}<\sum_{j=1}^{k}\left[\omega_{j} v_{i j}^{L}+m_{i j}\left(v_{i j}^{U}-v_{i j}^{L}\right)\right]\right\}$, then $I_{0} \cup I_{1} \cup I_{2}=M$.

Corollary 7. If $i \in I_{1}, d_{i}=1$; if $i \in I_{2}, d_{i}=-1$.

Proof. In Model (12), if $i \in I_{1}$ (i.e., $o_{i}>\sum_{j=1}^{k}\left[\omega_{j} v_{i j}^{L}+m_{i j}\left(v_{i j}^{U}-v_{i j}^{L}\right)\right]$ ), we have $a_{i}=0$ and $b_{i}>0$. According to the complementary slackness property, $d_{i}=1$. If $i \in I_{2}$, we prove that $d_{i}=-1$ in a similar fashion.

Theorem 10. Let $\omega_{j}^{*}, m_{i j}^{*}$ be the optimal solution to Model (12). The dual Model (26) is equivalent to:

$$
\begin{aligned}
& \operatorname{Max} \sum_{i=1}^{m}\left\{o_{i}-\sum_{j=1}^{k}\left[\omega_{j}^{*} v_{i j}^{L}+m_{i j}^{*}\left(v_{i j}^{U}-v_{i j}^{L}\right)\right]\right\} d_{i} \\
& \text { s.t. }\left\{\begin{array}{l}
\sum_{i=1}^{m}\left(v_{i 1}^{L} d_{i}+q_{i 1}\right)+x_{1} \leq 0 \\
\sum_{i=1}^{m}\left(v_{i j}^{L} d_{i}+q_{i j}\right)-x_{j-1}+x_{j} \leq 0, j=2, \ldots, k \\
\left(v_{i j}^{U}-v_{i j}^{L}\right) d_{i}-q_{i j} \leq 0, i \in M, j \in K \\
-1 \leq d_{i} \leq 1, i \in M \\
q_{i j}, x_{j} \geq 0, i \in M, j \in K
\end{array}\right.
\end{aligned}
$$

Proof. From Corollary 6, we have $\sum_{i=1}^{m} \sum_{j=1}^{k}\left[\omega_{j}^{*} v_{i j}^{L}+m_{i j}^{*}\left(v_{i j}^{U}-v_{i j}^{L}\right)\right] d_{i}+\sum_{j=1}^{k} d(j, \varepsilon) x_{j}=0$.

\section{Economic significance of MICPV models with abstentions}

This section focuses on the economic significance of Model (14), its dual Model (15) and its equivalence (18). The other two scenarios of the place weight constraints in the MICPV model given in Section 4.2 and 4.3 can be interpreted in a similar fashion.

\section{(5.1) Economic significance of the preferential voting models with abstentions}

- Without considering the moderator, the proposed preferential voting DEA model with abstentions focuses on obtaining the optimal aggregated scores for candidates under different constraints on place weights.

- By incorporating the moderator, the MICPV model with abstentions devotes to achieving the overall consensus between the moderator's expectation and all candidates' aggregate voting scores. At the same time, the model can also help to rank the candidates and select a winner.

\section{(5.2) Economic significance of dual variables}


- The dual variables in Model (15) are considered as the marginal value of the moderator's opinion.

The objective function of Model (15), $W=\sum_{i=1}^{m} o_{i} y_{i}$ can be interpreted as the overall optimal value of the moderator's opinions on all candidates. As $y_{i}=\frac{\partial W}{\partial o_{i}}$, it can be considered as the marginal value of the moderator's opinion on candidate $i$. This formulation reflects that the value of the moderator is accomplished by influencing voters' preferences. In other words, the presence of the moderator is able to influence the weighted average voting scores obtained by the candidates. Therefore, $y_{i}$ in (15) characterizes the marginal value of the moderator's opinion in changing candidate $i$ 's score, and the objective function in (15) gauges the overall influence of the moderator's opinion on all candidates' votes.

- The dual variables in Model (18) can be further interpreted as the marginal utility of the moderator's influence on candidate $i$ 's score.

Theorem 8 establishes the equivalence of Model (15) and Model (18), so the optimal solution $\omega_{j}^{*}, y_{i}^{*}, m_{i j}^{*}, \alpha_{i j}^{*}, i \in M, j \in K$ satisfy

$$
\begin{aligned}
\sum_{i=1}^{m} o_{i} y_{i}^{*} & =\sum_{i=1}^{m}\left\{o_{i}-\sum_{j=1}^{k}\left[\omega_{j}^{*} v_{i j}^{L}+m_{i j}^{*}\left(v_{i j}^{U}-v_{i j}^{L}\right)\right]\right\} y_{i}^{*} \\
& =\sum_{i=1}^{m}\left\{o_{i}-\sum_{j=1}^{k} \omega_{j}^{*}\left[v_{i j}^{L}+\alpha_{i j}^{*}\left(v_{i j}^{U}-v_{i j}^{L}\right)\right]\right\} y_{i}^{*}
\end{aligned}
$$

where $v_{i j}^{L}+\alpha_{i j}^{*}\left(v_{i j}^{U}-v_{i j}^{L}\right)$ denotes the optimal votes of candidate $i$ in place $j$ after accounting for abstentions, and $S_{i}\left(\omega_{j}^{*}, \alpha_{i j}^{*}\right)=\sum_{j=1}^{k} \omega_{j}^{*}\left[v_{i j}^{L}+\alpha_{i j}^{*}\left(v_{i j}^{U}-v_{i j}^{L}\right)\right]$ represents candidate $i$ 's optimal weighted voting score for all places. Mathematically, $o_{i}-\sum_{j=1}^{k} \omega_{j}^{*}\left[v_{i j}^{L}+\alpha_{i j}^{*}\left(v_{i j}^{U}-v_{i j}^{L}\right)\right]$ measures the deviation between the moderator's expectation for candidate $i$ and the candidate's performance in the voting, and $y_{i}$ is a partial derivative of $W, y_{i}=\frac{\partial W}{\partial\left\{o_{i}-\sum_{j=1}^{k}\left[\omega_{j} v_{i j}^{L}+m_{i j}\left(v_{i j}^{U}-v_{i j}^{L}\right)\right]\right\}}$. Consequently, $y_{i}$ can also be interpreted as the marginal utility of the moderator's influence on the candidate $i$ 's score $S_{i}\left(\omega_{j}, \alpha_{i j}\right)$, and $\sum_{i=1}^{m}\left\{o_{i}-\sum_{j=1}^{k}\left[\omega_{j}^{*} v_{i j}^{L}+m_{i j}^{*}\left(v_{i j}^{U}-v_{i j}^{L}\right)\right]\right\} y_{i}^{*}$ as the total utility of the moderator's influence on all candidates. Next we shall discuss the implications of this influence on different categories of candidates.

\section{(5.3) A partition of candidates based on the moderator's influence over the voters}


Since $\sum_{i=1}^{m} o_{i} y_{i}=\sum_{i=1}^{m}\left\{o_{i}-\sum_{j=1}^{k}\left[\omega_{j} v_{i j}^{L}+m_{i j}\left(v_{i j}^{U}-v_{i j}^{L}\right)\right]\right\} y_{i}$, we have

$$
\begin{aligned}
& \sum_{i=1}^{m} o_{i} y_{i} \\
= & \sum_{i \in I_{0}}\left\{o_{i}-\sum_{j=1}^{k}\left[\omega_{j} v_{i j}^{L}+m_{i j}\left(v_{i j}^{U}-v_{i j}^{L}\right)\right]\right\} y_{i} \quad(36-1) \\
+ & \sum_{i \in I_{1}}\left\{o_{i}-\sum_{j=1}^{k}\left[\omega_{j} v_{i j}^{L}+m_{i j}\left(v_{i j}^{U}-v_{i j}^{L}\right)\right]\right\} y_{i} \quad(36-2) \\
+ & \sum_{i \in I_{2}}\left\{o_{i}-\sum_{j=1}^{k}\left[\omega_{j} v_{i j}^{L}+m_{i j}\left(v_{i j}^{U}-v_{i j}^{L}\right)\right]\right\} y_{i} \quad(36-3)
\end{aligned}
$$

Let $I_{0}=\left\{i \mid o_{i} \triangleq \sum_{j=1}^{k}\left[\omega_{j} v_{i j}^{L}+m_{i j}\left(v_{i j}^{U}-v_{i j}^{L}\right)\right]\right\}, I_{1} \triangleq\left\{i \mid o_{i}>\sum_{j=1}^{k}\left[\omega_{j} v_{i j}^{L}+m_{i j}\left(v_{i j}^{U}-v_{i j}^{L}\right)\right]\right\}$, and $I_{2} \triangleq\left\{i \mid o_{i}<\sum_{j=1}^{k}\left[\omega_{j} v_{i j}^{L}+m_{i j}\left(v_{i j}^{U}-v_{i j}^{L}\right)\right]\right\}$, which are referred to as, from the moderator's perspective, consistent, underachieved, and overachieved candidates, respectively. Obviously, $I_{0} \cup I_{1} \cup I_{2}=M$.

(i) For an underachieved candidate $i \in I_{1}$, the moderator expects that he/she should receive more votes. When his/her uncertain votes $\left[v_{i j}^{L}, v_{i j}^{U}\right]$ are accounted for, as $m_{i j}=\omega_{j} \alpha_{i j}$, a larger $\alpha_{i j}$ brings up his/her score $\sum_{j=1}^{k}\left[\omega_{j} v_{i j}^{L}+m_{i j}\left(v_{i j}^{U}-v_{i j}^{L}\right)\right]$, thereby closing in the gap between the moderator's expectation and candidate $i$ 's performance. As per Corollary 3, $y_{i}=1>0$, we have $\alpha_{i j}=1$ by Property 1 . This indicates that, to minimize the deviation between $o_{i}$ and $\sum_{j=1}^{k}\left[\omega_{j} v_{i j}^{L}+m_{i j}\left(v_{i j}^{U}-v_{i j}^{L}\right)\right]$, all abstentions casted for underachieved candidate $i$ should be converted to approval votes, thereby achieving maximum consensus level between the moderator's expectation and the weighted voting score for this underachieved candidate $i$. The significance of the expression in $(36-2)$ is that, to maximize the influence of the moderator, all uncertain votes for those candidates whose voting scores underperform the moderator's expectations should be ascribed to approval votes.

(ii) For an overachieved candidate $i \in I_{2}$, the moderator does not think that he/she deserves such a high vote. In this case, if all uncertain votes $\left[v_{i j}^{L}, v_{i j}^{U}\right]$ are reallocated, since $m_{i j}=$ $\omega_{j} \alpha_{i j}$, a smaller $\alpha_{i j}$ brings down his/her score $\sum_{j=1}^{k}\left[\omega_{j} v_{i j}^{L}+m_{i j}\left(v_{i j}^{U}-v_{i j}^{L}\right)\right]$, thereby leading to a smaller deviation between the moderator's opinion and the voters' aggregate choice. Based on Corollary 3, $y_{i}=-1<0$, thus we have $\alpha_{i j}=0$ according to Property 2. This indicates that, to minimize the deviation between $o_{i}$ and $\sum_{j=1}^{k}\left[\omega_{j} v_{i j}^{L}+m_{i j}\left(v_{i j}^{U}-v_{i j}^{L}\right)\right]$, none of the uncertain votes for overachieved candidate $i$ should be converted to approvals. The significance of the expression in $(36-3)$ is that, to maximize the moderator's influence on the voting scores for candidates whose voting scores exceed expectation, none of the abstentions should be treated as approval votes. 
(iii) For consistent candidates $i \in I_{0}$, by properly adjusting the values of $\omega_{j}$ and $m_{i j}$ (or $\alpha_{i j}$ indirectly), the model is able to achieve complete consensus between the moderator's preference and the voters' collective choice. In this case, Model (15) helps to find the moderator's optimal marginal influence $y_{i} \in(-1,1), i \in I_{0}$ and to achieve a complete accord.

Thus, Eq. (36) demonstrates that the moderator's optimal influence over the voters can be partitioned into three categories as per the difference between his/her expectation and the voting scores of the candidates. Based on the specific category, the optimal marginal value of the moderator's opinion $y_{i}$ can be determined accordingly.

This result sheds structural insights into the role of the moderator in influencing the outcomes in a preferential voting. The moderator can use the statistics from polls as a proxy of the voters' collective choice and compare this information with his/her expectations. After assessing the differences, the moderator can devise optimal strategies to maximize his/her influence on voters: For overachieved candidates, the moderator can launch campaigns to undermine their positions; For underachieved candidates, the moderator has to work harder to promote them; For consistent candidates, our model helps the moderator to find the means to maintain the balance.

\section{Numerical examples}

This section provides different numerical examples to illustrate how the proposed models can be applied. First, we consider an example under the preferential voting DEA model with abstentions without accounting for a moderator's influence. Subsequently, various scenarios of the moderator's opinions are entertained to illustrate how the moderator influences the voting result differently. As illustrated by the numerical examples below, the original optimal solutions derived from the two models may result in fractional votes. We then employ the standard branch-and-bound method to find integer solutions for reallocated votes.

\subsection{A preferential voting DEA example with abstentions}

Assume that four candidates are competing for two places in a preferential election. The number of votes that candidate $i$ receives for place $j$ is denoted by $v_{i j}, i \in M=\{1,2,3,4\}, j \in K=\{1,2\}$ as shown in Table 1.

Table 1: A preferential voting DEA example with abstentions

\begin{tabular}{c|cc}
\hline \multirow{2}{*}{ Candidate $i$} & \multicolumn{2}{|c}{ Votes } \\
\cline { 2 - 3 } & $v_{i 1}$ & $v_{i 2}$ \\
\hline 1 & {$[2,6]$} & {$[3,8]$} \\
2 & {$[1,4]$} & {$[6,8]$} \\
3 & {$[6,8]$} & {$[0,2]$} \\
4 & {$[0,3]$} & {$[1,3]$} \\
\hline
\end{tabular}


In Table 1 , the interval value $[2,6]$ for candidate 1 at place 1 indicates that the candidate receives a minimum of two and a maximum of six votes placing him/her at No. 1. Other interval values can be interpreted similarly. By assuming $d(1, \varepsilon)=d(2, \varepsilon)=0$ in Model (3), the optimal solution for the four candidates is derived as shown in Table $2^{1}$.

Table 2: Results of the preferential voting DEA example with abstentions

\begin{tabular}{|c|c|c|c|c|c|c|c|c|}
\hline \multirow{2}{*}{ Candidate $i$} & \multicolumn{2}{|c|}{ Votes } & \multicolumn{4}{|c|}{ Optimal solutions } & \multirow{2}{*}{$\frac{\text { Maximum objective value }}{Z_{i}^{*}}$} & \multirow{2}{*}{ Ranking } \\
\hline & $v_{i 1}$ & $v_{i 2}$ & $\omega_{1}^{*}$ & $m_{i 1}^{*}$ & $\omega_{2}^{*}$ & $m_{i 2}^{*}$ & & \\
\hline 1 & 4.2768 & 6.6039 & 0.1149 & 0.0654 & 0.0770 & 0.0555 & 1 & 1 \\
\hline 2 & 3.0901 & 7.4572 & 0.1121 & 0.0781 & 0.0877 & 0.0639 & 1 & 1 \\
\hline 3 & 7.0863 & 1.5761 & 0.1274 & 0.0692 & 0.0618 & 0.0487 & 1 & 1 \\
\hline 4 & 3 & 3 & 0.1667 & 0.1667 & 0.1389 & 0.1389 & 0.9167 & 2 \\
\hline
\end{tabular}

The optimal solution in Table 2 fails to distinguish the priority of the first three candidates because their optimal scores are all 1. To increase the discrimination power, we shall apply Model (5) next, this helps to circumvent Model (3)'s pitfall of assigning the same score for multiple candidates. It also overcomes another weakness of the DEA voting model: for the same place, different weights are derived for different candidates.

Let $d(j, \varepsilon)=\varepsilon$. Plugging the input information in Table 1 into Model (5) yields the maximum objective value of 0.0833 .

The optimal solution leads to fractional votes for the candidates after abstentions are reallocated. To ensure that all candidates receive integer votes, we shall employ the branch and bound method for integer programming (Little et al., 1963). In this case, $v_{i j}=v_{i j}^{L}+\alpha_{i j}\left(v_{i j}^{U}-v_{i j}^{L}\right)$ and $\alpha_{i j}\left(v_{i j}^{U}-v_{i j}^{L}\right)$ must be integers. Because $\alpha_{i j}=\frac{m_{i j}}{w_{j}}$, we need to consider two branches $v_{i j}^{L}+\frac{m_{i j}}{w_{j}}\left(v_{i j}^{U}-v_{i j}^{L}\right) \leqslant\left\lfloor v_{i j}\right\rfloor$ and $v_{i j}^{L}+\frac{m_{i j}}{w_{j}}\left(v_{i j}^{U}-v_{i j}^{L}\right) \geqslant\left\lfloor v_{i j}\right\rfloor+1$, where $\left\lfloor v_{i j}\right\rfloor$ returns the integer part of $v_{i j}$. It is apparent that one and only one of these two branches is feasible, which will be added to the original model and the other infeasible branch will be discarded. This process is repeated for other decision variables progressively and sometimes multiple rounds are needed for the same decision variable in order to ensure integer votes for all candidates. Eventually, the candidates' final weighted voting scores $S_{i}\left(\omega_{j}^{*}, \alpha_{i j}^{*}\right)=\sum_{j=1}^{k} \omega_{j}^{*}\left[v_{i j}^{L}+\right.$ $\left.\alpha_{i j}^{*}\left(v_{i j}^{U}-v_{i j}^{L}\right)\right]$ are obtained and shown in Table 3. Without considering external influence from any moderator, candidate 3 arises as the winner.

Table 3: Final score of the preferential voting DEA example with abstentions

\begin{tabular}{c|cc|c|c}
\hline \multirow{2}{*}{ Candidate $i$} & \multicolumn{2}{|c|}{ Votes } & Score & \multirow{2}{*}{ Ranking } \\
\cline { 2 - 4 } & $v_{i 1}$ & $v_{i 2}$ & $S_{i}$ & \\
\hline 1 & 2 & 3 & 0.5833 & 4 \\
2 & 1 & 6 & 0.6665 & 3 \\
3 & 6 & 0 & 1 & 1 \\
4 & 3 & 3 & 0.75 & 2 \\
\hline
\end{tabular}

\footnotetext{
${ }^{1}$ The result is obtained by using Matlab R2013a
} 


\subsection{An MICPV example with abstentions}

The basic setting of this example is the same as that in Section 6.1 except that the moderator's opinions are listed in the last column in Table 4.

Table 4: An MICPV example with abstentions

\begin{tabular}{c|cc|c}
\hline \multirow{2}{*}{ Candidate $i$} & \multicolumn{2}{|c|}{ Votes } & Moderator's Opinion \\
\cline { 2 - 4 } & $v_{i 1}$ & $v_{i 2}$ & $o_{i}$ \\
\hline 1 & {$[2,6]$} & {$[3,8]$} & 3 \\
2 & {$[1,4]$} & {$[6,8]$} & 7 \\
3 & {$[6,8]$} & {$[0,2]$} & 6 \\
4 & {$[0,3]$} & {$[1,3]$} & 5 \\
\hline
\end{tabular}

Assume that $d(1, \varepsilon)=d(2, \varepsilon)=0.5$ and $m_{i j}=\omega_{j} \alpha_{i j}, i=1,2,3,4, j=1,2$, plugging the input information in Table 4 into Model (11) leads to the result in Table 5.

Table 5: Final scores of the MICPV example with abstentions

\begin{tabular}{c|cc|c|c|c}
\hline \multirow{2}{*}{ Candidate $i$} & \multicolumn{2}{|c|}{ Votes } & Moderator's Opinion & Score & \multirow{2}{*}{ Ranking } \\
\cline { 2 - 6 } & $v_{i 1}$ & $v_{i 2}$ & $o_{i}$ & $S_{i}$ & \\
\hline 1 & 2 & 3 & 3 & 3.5 & 4 \\
2 & 3.1486 & 7.7028 & 7 & 7 & 1 \\
3 & 6 & 0 & 6 & 6 & 2 \\
4 & 3 & 3 & 5 & 4.5 & 3 \\
\hline
\end{tabular}

Furthermore, there exists the following relationship between the optimal solution of the primal model and that of the dual model,

$$
\begin{aligned}
& \left(2 \omega_{1}^{*}+4 m_{11}^{*}+3 \omega_{2}^{*}+5 m_{12}^{*}\right) d_{1}^{*}+\left(\omega_{1}^{*}+3 m_{21}^{*}+6 \omega_{2}^{*}+2 m_{22}^{*}\right) d_{2}^{*} \\
+ & \left(6 \omega_{1}^{*}+2 m_{31}^{*}+0 \omega_{2}^{*}+2 m_{32}^{*}\right) d_{3}^{*}+\left(0 \omega_{1}^{*}+3 m_{41}^{*}+\omega_{2}^{*}+2 m_{42}^{*}\right) d_{4}^{*}+0.5 * x_{1}^{*}+0.5 * x_{2}^{*} \\
= & 0
\end{aligned}
$$

Eq. (37) verifies Corollary 6. Furthermore, as per Theorem 10, the objective function is equivalent to

$$
\begin{aligned}
& \operatorname{Max} D_{0}=\left[3-\left(2 \omega_{1}^{*}+4 m_{11}^{*}+3 \omega_{2}^{*}+5 m_{12}^{*}\right)\right] d_{1}+\left[7-\left(\omega_{1}^{*}+3 m_{21}^{*}+6 \omega_{2}^{*}+2 m_{22}^{*}\right)\right] d_{2} \\
+ & {\left[6-\left(6 \omega_{1}^{*}+2 m_{31}^{*}+0 \omega_{2}^{*}+2 m_{32}^{*}\right)\right] d_{3}+\left[5-\left(0 \omega_{1}^{*}+3 m_{41}^{*}+\omega_{2}^{*}+2 m_{42}^{*}\right)\right] d_{4} }
\end{aligned}
$$

Under a moderator's influence, Eq. (38) clearly illustrates the role of the moderator: his/her optimal value is reflected in the aggregate utility of the deviation between the moderator's opinion and the candidates' voting scores.

Analytic results in Table 5 provide us an example to understand the economic significance of Corollary 7. First, the voting score of candidate 1 is 3.5 and exceeds the moderator's expectation $o_{1}=3$. In this case, the corresponding dual variable $d_{1}^{*}=-1$, implying that none of the abstentions casted for candidate 1 will be counted as approval votes. Conversely, the voting score of candidate 
4 comes in at 4.5 , below the moderator's expectation of $o_{4}=5$. As such, the dual variable $d_{4}^{*}=1$, meaning that all abstentions for candidate 4 will be treated as approval votes. The aforesaid treatment of abstentions is able to maximize the moderator's influence over the voters and achieve the maximum consensus level (or the minimum deviation) between his/her expectation and the voters' collective choice.

Table 5 indicates that candidate 2 receives fractional votes, which should be integers. Therefore, we follow the same branch-and-bound procedure as mentioned in Section 6.1 to find integer solutions. The resulting votes of candidates $v_{i j}, i=1,2,3,4, j=1,2$, and their final scores $S_{i}$ are shown in Table 6 .

Table 6: Final integer scores of the MICPV example

\begin{tabular}{c|cc|c|c|c}
\hline \multirow{2}{*}{ Candidate $i$} & \multicolumn{2}{|c|}{ Votes } & Moderator's Opinion & Score & \multirow{2}{*}{ Ranking } \\
\cline { 2 - 5 } & $v_{i 1}$ & $v_{i 2}$ & $o_{i}$ & $S_{i}$ & \\
\hline 1 & 2 & 3 & 3 & 3.5 & 4 \\
2 & 4 & 6 & 7 & 7 & 1 \\
3 & 6 & 0 & 6 & 6 & 2 \\
4 & 3 & 3 & 5 & 4.5 & 3 \\
\hline
\end{tabular}

Comparing the ranking results in Table 3 (without a moderator) and Table 6 (with a moderator), one can easily see that the candidates' final scores are influenced by the moderator's opinions. Under this influence, the candidates' scores are closer to the moderator's opinions if a complete consensus is not achievable. In this example, candidate 2 arises as the winner as opposed to candidate 3 in the previous example without a moderator.

\subsection{Three cases about the moderator's influence on the voting result}

To better illustrate how the moderator's opinions influence the voting result, we employ the raw voting data in Table 1 and apply the same computation process in Section 6.2. By experimenting with different scenarios of the moderator's expected scores on candidates, we obtain the following three cases (see Table 7 and Figure 1).

Table 7: Three cases about the moderator's influence on the voting result

\begin{tabular}{c|cc|cc|cr}
\hline \multirow{2}{*}{ Candidate $i$} & \multicolumn{2}{|c|}{ Case 1 } & \multicolumn{2}{|c|}{ Case 2 } & \multicolumn{2}{c}{ Case 3 } \\
\cline { 2 - 7 } & $o_{i}$ & $S_{i}$ & $o_{i}$ & $S_{i}$ & $o_{i}$ & $S_{i}$ \\
\hline 1 & 5 & 4.9998 & 3 & 3.5 & 6 & 5.9997 \\
2 & 7 & 6.9996 & 4 & 4 & 4 & 4 \\
3 & 6 & 6.3942 & 6 & 6 & 2 & 6 \\
4 & 5 & 4.8942 & 9 & 4.5 & 9 & 4.5 \\
\hline
\end{tabular}

Case 1 Fig. 1-1 demonstrates a case that the moderator's expectations are attainable by influencing the voters' decision, resulting in two fairly close curves between the moderator's opinion and the output based on our proposed model. 

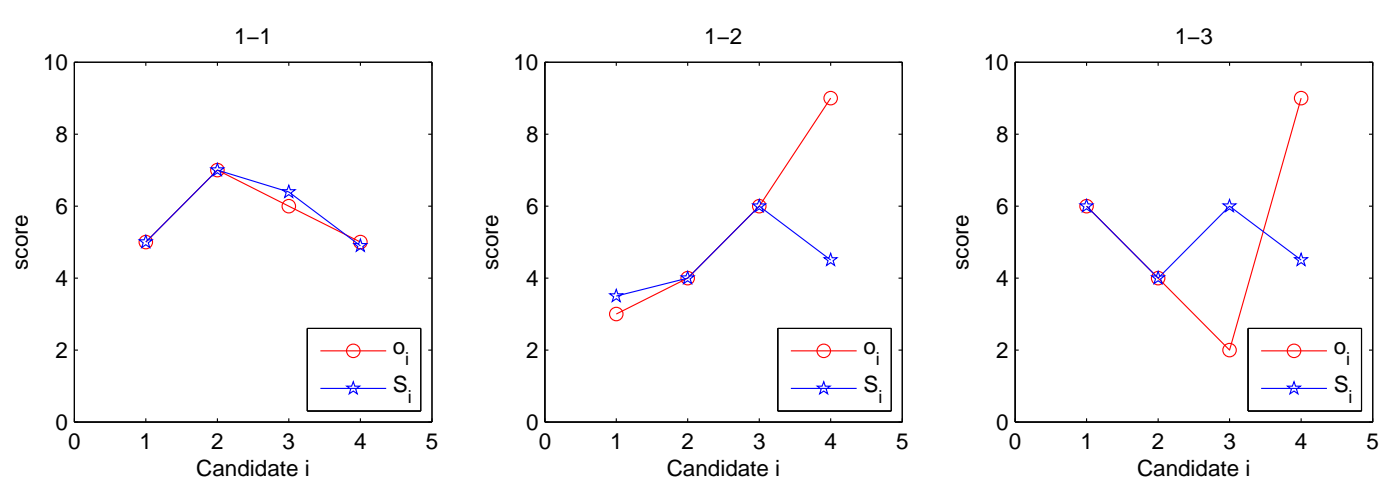

Figure 1: The difference between the moderator's opinions and candidates' final scores

Case 2 Fig. 1-2 illustrates a scenario that the moderator's most favoured candidate 4 (with the highest expected score of 9) cannot rise to the top after abstentions are reallocated. This is due to the fact that candidate 4 receives the lowest raw votes (a maximum of 3 votes in the first and second place, respectively). After the moderator exercises his/her influence, the weighted voting score for candidate 4 rises to the second place, but can never reach the top given the voters' choice. For the other three candidates, consensus can by and large be reached between the moderator and the voters.

Case 3 Fig. 1-3 displays another situation that the moderator's opinion on the most and least preferred candidates differs from the collective choice of the voters as reflected in their raw votes in Table 1. As the moderator least prefers candidate 3 , he/she receives none of the uncertain votes. However, this cannot prevent candidate 3 from receiving the highest weighted score given the voters' strong support (candidate 3 obtains a minimum of 6 votes in the first place). On the other hand, similar to Case 2, the moderator can influence abstention votes to increase his/her most preferred candidate 4's weighted score, but the final score is still subject to the voters' collective choice as reflected in their raw votes.

In summary, the previous experiment indicates that the moderator's opinion plays an important role in influencing abstention votes to reach weighted vote scores that are closer to the moderator's expectations. However, this influence can by no means override the voters' choice. If the moderator's expectation is unreasonably high or low for a particular candidate (for instance, candidate 3 or 4 in Case 3), while his/her influence can increase or decrease the candidate's weighted vote score, the final score is contingent upon the voters' choice and differs from the moderator's opinion.

\section{Conclusions}

In a preferential election, invalid ballots or abstentions typically represent the voters' uncertainty about their choice. Whether abstentions are counted towards approval or disapproval votes can dramatically change the election result. This paper first generalizes Cook and Kress (1990)'s DEA model 
for aggregating preference rankings by allowing voting scores to assume interval values so that abstentions can be explicitly accommodated. Subsequently, an MICPV model with abstentions is proposed to incorporate opinion leaders as a moderator. This moderator can exploit his/her resources to influence voters' decision so that his expectations about the voting result can be achieved or approximated as much as possible. Different versions of the model are discussed given different types of place weight constraints. Economic interpretations of our results show how the moderator exerts his/her influence on the voters. The key features of the proposed models are as follows:

- Invalid ballots and abstentions are collectively treated as abstentions and interval values are employed to characterize voters' inherent uncertainty about the voting.

- A preferential voting DEA model with abstentions is proposed.

- A moderator is introduced into an MICPV model to represent opinion leaders/brokers whose preferences are reflected in his/her expected voting scores for candidates. The objective function is to maximize the consensus level (or minimize the deviation) between his/her expectations and the voters' collective choice over the candidates.

- The proposed MICPV model and its variants incorporate different scenarios of place weight constraints to enhance their applicability to diverse preferential election settings.

- Analytic results reveal the properties of the primal-dual variables in the MICPV models as well as their economic interpretations.

The main contributions of this paper are as follows:

- Given that the DEA method is widely applied to evaluate relative efficiency of decision making units, the proposed preferential voting DEA model with abstentions can optimally distribute uncertain votes and, then, make better use of available voting information. Moreover, place weights are objectively determined as the optimal solution to the DEA voting model, providing a fair overall assessment of candidates' rank in a preferential election with abstentions.

- The MICPV model with abstentions and its variants not only respect voters' choice, but also consider the moderator's influence. The proposed models can also provide an optimal allocation of voting resources from a consensus angle. Key managerial insights of the proposed MICPV model and its variants are to furnish viable campaign strategies for opinion leaders in an election: By using the poll statistics as reference points, they can devise suitable strategies to promote their preferred candidates and/or undermine their non-preferred candidates, thereby exercising the maximum influence over the voters and achieving desired voting results. 


\section{References}

\section{References}

Altuzarra, A., Moreno-Jiménez, J. M., \& Salvador, M. (2010). Consensus building in AHP-group decision making: A Bayesian approach. Operations Research, 58(6), 1755-1773.

Angiz, M. Z., Tajaddini, A., Mustafa, A., \& Kamali, M. J. (2012). Ranking alternatives in a preferential voting system using fuzzy concepts and data envelopment analysis. Computers \& Industrial Engineering, 63(4), 784-790.

Arrow, K. J. (1950). A difficulty in the concept of social welfare. The Journal of Political Economy, 58, 328-346.

Ben-Arieh, D., \& Easton, T. (2007). Multi-criteria group consensus under linear cost opinion elasticity. Decision Support Systems, 43(3), 713-721.

Beruvides, M. G. (1995). Group decision support systems and consensus building: Issues in electronic media. Computers \& Industrial Engineering, 29(1-4), 601-605.

Borda, J. C. (1784). Memoire sur les elections au scrutin, Histoire de l'Academie Royale de Science.

Bouton, L., Castanheira, M., \& Llorente-Saguer, A. (2015). Divided majority and information aggregation: Theory and experiment. Journal of Public Economics, 134, 114-128.

Burnett, C. M., \& Kogan, V. (2015). Ballot (and voter) "exhaustion" under Instant Runoff Voting: An examination of four ranked-choice elections. Electoral Studies, 37, 41-49.

Büyüközkan, G., \& Güleryüz, S. (2016). A new integrated intuitionistic fuzzy group decision making approach for product development partner selection. Computers \& Industrial Engineering, 102, 383-395.

Chandrasekher, M. (2015). Dynamically consistent voting rules. Journal of Economic Theory, 160, 175-187.

Chen, X., Zhang, H., \& Dong, Y. (2015). The fusion process with heterogeneous preference structures in group decision making: a survey. Information Fusion, 24, 72-83.

Cook, W. D., \& Kress, M. (1990). A data envelopment model for aggregating preference rankings. Management Science, 36(11), 1302-1310.

Cook, W. D., Kress, M., \& Seiford, L. M. (1997). A general framework for distance-based consensus in ordinal ranking models. European Journal of Operational Research, 96(2), 392-397.

Dong, Y., Liu, Y., Liang, H., Chiclana, F., \& Herrera-Viedma, E. (2018). Strategic weight manipulation in multiple attribute decision making. Omega, 75, 154-164.

Dong, Y., Ding, Z., Martínez, L., \& Herrera, F. (2017). Managing consensus based on leadership in opinion dynamics. Information Sciences, 397-398, 187-205.

Ebrahimnejad, A., Tavana, M., \& Santos-Arteaga, F. J. (2016). An integrated data envelopment analysis and simulation method for group consensus ranking. Mathematics and Computers in Simulation, 119, 1-17.

Fishburn, P. C. (1970). Arrow's impossibility theorem: Concise proof and infinite voters. Journal of Economic Theory, 2(1), 103-106.

Franceschini, F., \& Maisano, D. (2015). Checking the consistency of the solution in ordinal semi-democratic decision-making problems. Omega, 57, 188-195.

Gans, J. S., \& Smart, M. (1996). Majority voting with single-crossing preferences. Journal of public Economics, 59(2), 219-237. 
Geng, X., Gong, X., \& Chu, X. (2016). Component oriented remanufacturing decision-making for complex product using dea and interval 2-tuple linguistic topsis. International Journal of Computational Intelligence Systems, 9(5), 984-1000.

Gong, Z. W., \& Wang, L. H. (2017). On consistency test method of expert opinion in ecological security assessment. International Journal of Environmental Research \& Public Health, 14(9), 1012.

Gong, Z. W., Xu, X. X., Zhang, H. H., Ozturk, U. A., Herrera-Viedma, E., \& Xu, C. (2015a). The consensus models with interval preference opinions and their economic interpretation. Omega, 55, 81-90.

Gong, Z. W., Zhang, H. H., Forrest, J., Li, L. S., \& Xu, X. X. (2015b). Two consensus models based on the minimum cost and maximum return regarding either all individuals or one individual. European Journal of Operational Research, 240(1), 183-192.

González-Pachón, J., \& Romero, C. (1999). Distance-based consensus methods: a goal programming approach. Omega, 27, 341-347.

Herrera-Viedma, E., Alonso, S., Chiclana, F., \& Herrera, F. (2007). A consensus model for group decision making with incomplete fuzzy preference relations. IEEE Transactions on Fuzzy Systems, 15(5), 863-877.

Hummel, P. (2011). Information aggregation in multicandidate elections under plurality rule and runoff voting. Mathematical Social Sciences, 62(1), 1-6.

Hwang, C. L., \& Lin, M. J. (2012). Group decision making under multiple criteria: methods and applications. Berlin Heidelberg: Springer-Verlag.

Janis, I. L. (1972). Victims of groupthink: a psychological study of foreign-policy decisions and fiascoes.

Kahneman, D., \& Tversky, A. (1979). Prospect theory: An analysis of decision under risk. Econometrica: Journal of the Econometric Society, 47(2), 263-291.

Labella, Á., Liu, Y., Rodríguez, R. M., \& Martínez, L. (2017). Analyzing the performance of classical consensus models in large scale group decision making: a comparative study. Applied Soft Computing, DOI: 10.1016/j.asoc.2017.05.045.

Little, J. D., Murty, K. G., Sweeney, D. W., \& Karel, C. (1963). An algorithm for the traveling salesman problem. Operations research, 11(6), 972-989.

Liu, Y., Liang, C., Chiclana, F., \& Wu, J. (2017). A trust induced recommendation mechanism for reaching consensus in group decision making. Knowledge-Based Systems, 119, 221-231.

Llamazares, B., \& Pen̈a, T. (2009). Preference aggregation and DEA: An analysis of the methods proposed to discriminate efficient candidates. European Journal of Operational Research, 197(2), 714-721.

Meng, F. Y., Tan, C. Q., \& Chen, X. H. (2016). Multiplicative consistency analysis for interval fuzzy preference relations: A comparative study. Omega, 68.

Myers, D. G., \& Lamm, H. (1975). The polarizing effect of group discussion: The discovery that discussion tends to enhance the average prediscussion tendency has stimulated new insights about the nature of group influence. American Scientist, 63(3), 297-303.

Oukil, A., \& Amin, G. R. (2015). Maximum appreciative cross-efficiency in DEA: A new ranking method. Computers \& Industrial Engineering, 81, 14-21.

Palomares, I., Estrella, F. J., Martínez, L., \& Herrera, F. (2014). Consensus under a fuzzy context: Taxonomy, analysis framework AFRYCA and experimental case of study. Information Fusion, 20(15), 252-271.

Power, T. J., \& Roberts, J. T. (1995). Compulsory voting, invalid ballots, and abstention in Brazil. Political 
Research Quarterly, 48(3), 795-826.

Procaccia, A. D., Shah, N., \& Zick, Y. (2016). Voting rules as error-correcting codes. Artificial Intelligence, 231, $1-16$.

Simon, H. A. (1991). Bounded rationality and organizational learning. Organization science, 2(1), 125-134.

Sivagami, V. M., Revathi, N., \& Sumathi, D. G. (2011). Votegrid: a mobile ballot system for decision making in grid environment. Procedia Computer Science, 3, 287-291.

Stoner, J. A. F. (1961). A comparison of individual and group decisions involving risk. Massachusetts Institute of Technology.

Sun, B. Z., \& Ma, W. M. (2015). An approach to consensus measurement of linguistic preference relations in multi-attribute group decision making and application. Omega, 51, 83-92.

Tan, X., Gong, Z., Huang, M., \& Wang, Z. J. (2017a). Selecting cooking methods to decrease persistent organic pollutant concentrations in food of animal origin using a consensus decision-making model. International Journal of Environmental Research \& Public Health, 14(2), 187.

Tan, X., Gong, Z., Chiclana, F., \& Zhang, N. (2017b). Consensus modeling with cost chance constraint under uncertainty opinions. Applied Soft Computing, DOI: 10.1016/j.asoc.2017.08.049.

Tavares, L. V. (2012). An acyclic outranking model to support group decision making within organizations. Omega, 40, 782-790.

Wang, L., \& Gong, Z. (2017). Priority of a hesitant fuzzy linguistic preference relation with a normal distribution in meteorological disaster risk assessment. International Journal of Environmental Research \& Public Health, 14(10), 1203.

Wang, Y. M., Luo, Y., \& Hua, Z. (2007). Aggregating preference rankings using OWA operator weights. Information Sciences, 177(16), 3356-3363.

Wang, Y. M., Yang, J. B., \& Xu, D. L. (2005). A preference aggregation method through the estimation of utility intervals. Computers \& Operations Research, 32(8), 2027-2049.

Wu, J., Dai, L., Chiclana, F., Fujita, H., \& Herrera-Viedma, E. (2018). A minimum adjustment cost feedback mechanism based consensus model for group decision making under social network with distributed linguistic trust. Information Fusion, 41, 232-242.

Wu, J., Chiclana, F., Fujita, H., \& Herrera-Viedma, E. (2017). A visual interaction consensus model for social network group decision making with trust propagation. Knowledge-Based Systems, 122, 39-50.

Wu, Z. B., \& Xu, J. P. (2015). Managing consistency and consensus in group decision making with hesitant fuzzy linguistic preference relations. Omega, 65(3), 28-40.

Zhang, N., Gong, Z. W., \& Chiclana, F. (2017). Minimum cost consensus models based on random opinions. Expert Systems with Applications, 89, 149-159. 